\title{
Suppression of APOBEC3-mediated restriction of HIV-1 by Vif
}

\section{Yuqing Feng, Tayyba T. Baig, Robin P. Love and Linda Chelico*}

Department of Microbiology and Immunology, College of Medicine, University of Saskatchewan, Saskatoon, SK, Canada

\section{Edited by:}

Nadine Laguette, Centre National de la Recherche Scientifique, France

\section{Reviewed by:}

Atsushi Koito, Kumamoto University, Japan

Yoshitaka Sato, Nagoya University Graduate School of Medicine, Japan

\section{*Correspondence:}

Linda Chelico, Department of Microbiology and Immunology, College of Medicine, University of Saskatchewan, 107 Wiggins Road, Saskatoon, SK S7N 5E5, Canada e-mail: linda.chelico@usask.ca
The APOBEC3 restriction factors are a family of deoxycytidine deaminases that are able to suppress replication of viruses with a single-stranded DNA intermediate by inducing mutagenesis and functional inactivation of the virus. Of the seven human APOBEC3 enzymes, only APOBEC3-D, - F, -G, and -H appear relevant to restriction of HIV-1 in CD4+ T cells and will be the focus of this review. The restriction of HIV-1 occurs most potently in the absence of HIV-1 Vif that induces polyubiquitination and degradation of APOBEC3 enzymes through the proteasome pathway. To restrict HIV-1, APOBEC3 enzymes must be encapsidated into budding virions. Upon infection of the target cell during reverse transcription of the HIV-1 RNA into (-)DNA, APOBEC3 enzymes deaminate cytosines to form uracils in single-stranded (-)DNA regions. Upon replication of the (-)DNA to (+)DNA, the HIV-1 reverse transcriptase incorporates adenines opposite to the uracils thereby inducing $\mathrm{C} / \mathrm{G}$ to T/A mutations that can functionally inactivate HIV-1. APOBEC3G is the most studied APOBEC3 enzyme and it is known that Vif attempts to thwart APOBEC3 function not only by inducing its proteasomal degradation but also by several degradationindependent mechanisms, such as inhibiting APOBEC3G virion encapsidation, mRNA translation, and for those APOBEC3G molecules that still become virion encapsidated, Vif can inhibit APOBEC3G mutagenic activity. Although most Vif variants can induce efficient degradation of APOBEC3-D, $-F$, and $-G$, there appears to be differential sensitivity to Vifmediated degradation for APOBEC3H. This review examines APOBEC3-mediated HIV restriction mechanisms, how Vif acts as a substrate receptor for a Cullin5 ubiquitin ligase complex to induce degradation of APOBEC3s, and the determinants and functional consequences of the APOBEC3 and Vif interaction from a biological and biochemical perspective.

Keywords: HIV, mutagenesis, deaminase, Vif, APOBEC3, restriction factor, ubiquitination

\section{OVERVIEW}

Retrotransposons and endogenous retroviruses have been genomic parasites in organisms throughout evolution and have contributed to both species evolution and disease (Hancks and Kazazian, 2012). The APOBEC (Apolipoprotein B mRNA-editing enzyme-catalytic polypeptide) family of enzymes present in their earliest form in bony fish acted as a defense to retroelements (MacDuff et al., 2009). Due to expansion of retroelements through evolution, there was a corresponding expansion in the APOBEC family (Jern and Coffin, 2008; LaRue etal., 2008). The most recent expansion in placental mammals formed the APOBEClike 3 (APOBEC3) family in response to ancient pathogenic retroviruses (LaRue et al., 2008; Munk et al., 2012). Humans contain seven APOBEC3 (A3) enzymes (A3A, A3B, A3C, A3D, A3F, A3G, and A3H, Jarmuz et al., 2002; LaRue et al., 2009). The A3 enzymes act as host restriction factors to inhibit retroelement replication through either RNA binding ability or activity as single-stranded (ss) DNA cytosine deaminases that catalyze the formation of promutagenic uracils (Esnault et al., 2005; Bogerd etal., 2006; Chiu etal., 2006; Jonsson et al., 2006; Armitage etal., 2008; OhAinle etal., 2008; Khatua etal., 2010; Duggal etal., 2011; Koyama etal., 2013). Currently, A3 enzymes are primarily studied for their ability to restrict the replication of retroviruses (such as HIV-1, Sheehy et al., 2002; Harris etal., 2003; Mangeat etal., 2003; Zhang etal., 2003; Liddament et al., 2004; Wiegand et al., 2004; Zheng et al., 2004; Dang et al., 2006, 2008; OhAinle et al., 2008; Richardson et al., 2014) and other viruses with an ssDNA intermediate (such as Hepatitis B Virus, Kock and Blum, 2008; Lucifora et al., 2014). Restriction of the replication of these present day viruses occurs primarily through the deoxycytidine deamination activity of $\mathrm{A} 3$ enzymes which results in hypermutated and inactivated viral genomes. The gene duplications that resulted in the human A3 repertoire formed two general groups of deaminases with different Zinc (Z) coordinating domains: $\mathrm{A} 3 \mathrm{~A}, \mathrm{~A} 3 \mathrm{C}$, and $\mathrm{A} 3 \mathrm{H}$ are enzymes with a single $\mathrm{Z}$-domain and $\mathrm{A} 3 \mathrm{~B}, \mathrm{~A} 3 \mathrm{G}, \mathrm{A} 3 \mathrm{D}$, and $\mathrm{A} 3 \mathrm{~F}$ enzymes with two Zdomains (LaRue et al., 2008, Figure 1). For A3 enzymes with two Z-domains, only one domain is catalytically active, except for A3B, which may have two catalytically active domains (Hache et al., 2005; Navarro et al., 2005; Bogerd et al., 2007; Bonvin and Greeve, 2007, Figure 1).

For HIV-1 (referred to as HIV) to successfully infect humans, it must overcome numerous physical and immunological barriers (Harris et al., 2012; Shaw and Hunter, 2012; Xu et al., 2013). Within 


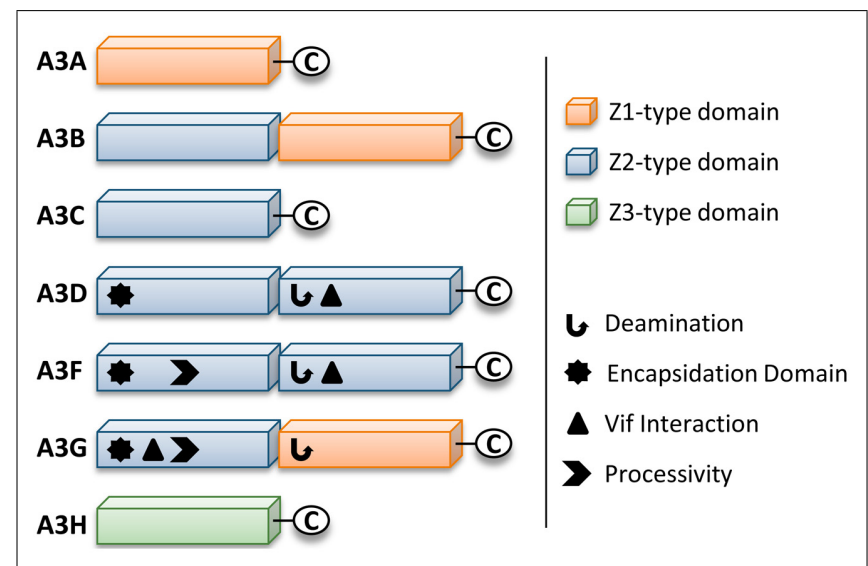

FIGURE 1 | Zinc (Z) coordinating-type domains of human $A 3$ enzymes. A3 enzymes coordinate zinc through the motif $\mathrm{H}-\mathrm{X}-\mathrm{E}-\mathrm{X}_{23}-28-\mathrm{P}-\mathrm{C}-\mathrm{X}_{2-4}-\mathrm{C}$. The glutamate activates a water molecule to enable zinc-hydroxidemediated nucleophilic attack to complete the deamination reaction. Deamination activity has been demonstrated for all $A 3$ enzymes. For the enzymes with two Z-type domains that restrict HIV in CD4+ T cells (A3D, $A 3 F$, and $A 3 G)$, a legend depicts known biochemical functions of each Z-type domain. A common feature of $\mathrm{A} 3$ enzymes with two Z-type domains is the segregation of functions in the N-terminal domain (NTD) and C-terminal domain (CTD). The NTD is responsible for encapsidation and the CTD is responsible for deamination activity. Both domains can bind nucleic acids. The binding site of Vif is in the NTD for A3G and in the CTD for A3D and $A 3 F$. The determinants for enzyme processivity have only been studied for A3G and A3F. A3G and A3F processivity is imparted by the NTD.

cells, HIV must overcome a network of restriction factors that are able to block specific replication steps of the virus, including A3 enzymes (Harris et al., 2012; Rahm and Telenti, 2012). HIV can overcome these restriction factors through mutations or encoding accessory proteins that specifically block the restriction factor function (Harris et al., 2012; Rahm and Telenti, 2012). HIV uses the viral infectivity factor (Vif) to overcome A3 enzymes (Sheehy et al., 2002; Conticello et al., 2003; Harris et al., 2003; Mangeat et al., 2003; Mariani et al., 2003). The Vif protein of simian immunodeficiency virus (SIV), the non-human primate form of the virus, has co-evolved with species-specific A3s for millions of years (Compton and Emerman, 2013). The HIV-1 predecessor, $\mathrm{SIV}_{\mathrm{cpz}}$ from chimpanzees underwent a key evolutionary event that altered the $3^{\prime}$ region of the vif gene that was essential for antagonism of human A3 function, along with further evolutionary changes in chimpanzees that adapted SIV $_{\mathrm{cpz}}$ for improved infection of humans (Etienne et al., 2013). To antagonize A3 enzymes, HIV Vif must maintain the ability to physically interact with relevant $\mathrm{A} 3 \mathrm{~s}$, host protein $\mathrm{CBF} \beta$ for stability (Jager et al., 2012; Zhang et al., 2012), and components of the host ubiquitin ligase assembly (Yu et al., 2003, 2004; Kobayashi et al., 2005; Xiao et al., 2006). Ultimately Vif thwarts A3s by inducing their polyubiquitination and degradation through the proteasome (Figures $\mathbf{2 A , B}$ ). It is thought that by disrupting the Vif-host cell interactions through novel pharmaceuticals, A3 enzymes can be used to suppress HIV. However, the natural balance of A3 enzymes and HIV must be first understood since there is evidence that HIV can take advantage of A3 enzymes to accelerate its quasispecies evolution (Simon et al., 2005; Kim et al., 2010).
From cell culture studies, it appears that only A3A, A3D, $\mathrm{A} 3 \mathrm{~F}, \mathrm{~A} 3 \mathrm{G}$, and $\mathrm{A} 3 \mathrm{H}$ are relevant to $\mathrm{HIV}$ restriction (Berger et al., 2011; Hultquist et al., 2011; Koning et al., 2011; Refsland et al., 2012; Chaipan et al., 2013). It is not surprising that not all seven A3 members restrict HIV replication since they likely evolved to restrict different retroelement pathogens (LaRue et al., 2008). There are two paradigms of how A3 enzymes can suppress HIV. A3A present in HIV target myeloid cells can restrict replication of incoming virions through low levels of deamination and possibility another mechanism that is not yet fully elucidated (Berger et al., 2011; Koning et al., 2011). In CD4+ T cells, A3D, A3F, $\mathrm{A} 3 \mathrm{G}$, and $\mathrm{A} 3 \mathrm{H}$ restrict $\mathrm{HIV} \Delta$ vif by becoming virion encapsidated in the HIV producer cell and traveling with the virion to the next susceptible cell where they catalyze promutagenic deaminations of cytosine to uracil in nascent single-stranded HIV (-)DNA (Hultquist et al., 2011, Figure 2A). Although A3B can also restrict HIV in this manner in 293T or HeLa cells, it is unable to become virion packaged and restrict HIV in $\mathrm{T}$ cell lines, has low expression in activated $\mathrm{T}$ cells, and is not antagonized by Vif, suggesting that restriction by $\mathrm{A} 3 \mathrm{~B}$ is not physiologically relevant (Doehle et al., 2005; Koning et al., 2009; Refsland et al., 2010; Hultquist et al., 2011; Pak et al., 2011). This review will focus on the restriction of HIV by virion encapsidated A3D, A3F, A3G, and A3H and how Vif antagonizes their function.

\section{A3-MEDIATED RESTRICTION OF HIV}

The A3D, A3F, A3G, and A3H molecules that escape Vif-mediated inhibition can restrict HIV by entering the assembling virus particle by binding RNA (HIV genome or cellular RNA such as 7SL or Y) that also interacts with the nucleocapsid (NC) portion of the Gag polyprotein (Alce and Popik, 2004; Cen et al., 2004; Douaisi et al., 2004; Svarovskaia et al., 2004; Burnett and Spearman, 2007; Khan et al., 2007; Bach et al., 2008; Bogerd and Cullen, 2008; Strebel and Khan, 2008; Wang et al., 2008; Ooms et al., 2010; Zhen et al., 2012, Figure 2A). After the virus enters the next target cell A3 enzymes exert their anti-viral function during the reverse transcription process (Suspene et al., 2004; Yu et al., 2004; Zheng et al., 2004; Dang et al., 2006; OhAinle et al., 2008, Figures 2A,C). Although A3D, A3F, A3G, and A3H are localized to the cytoplasm they require encapsidation to restrict HIV and are not able to access the (-)DNA of an incoming virus (Hultquist et al., 2011, Figure 2A). This may be due to the HIV capsid structure or that A3 enzymes can reside in regions of RNA processing, e.g., stress granules or P-bodies, where they may have a role in sequestering human retrotransposon RNA to prevent transposition (Chiu et al., 2006; Kozak et al., 2006; Stopak et al., 2007; Gallois-Montbrun et al., 2008). Since A3 enzymes are ssDNA deaminases, deamination activity is restricted to the (-)DNA strand (Yu et al., 2004, Figure 2C). The cytosine $(\mathrm{C}) \longrightarrow$ uracil $(\mathrm{U})$ deaminations catalyzed on the $(-)$ DNA strand become guanine $(\mathrm{G}) \longrightarrow$ adenine $(\mathrm{A})$ mutations when reverse transcriptase (RT) uses $\mathrm{U}$ as a template during (+)DNA strand synthesis (Yu et al., 2004, Figure 2C). The resulting "hypermutation" of the provirus leads to inactivation of HIV (Harris et al., 2003; Mangeat et al., 2003; Zhang et al., 2003, Figure 2A). Although it is known that many proviral genomes 


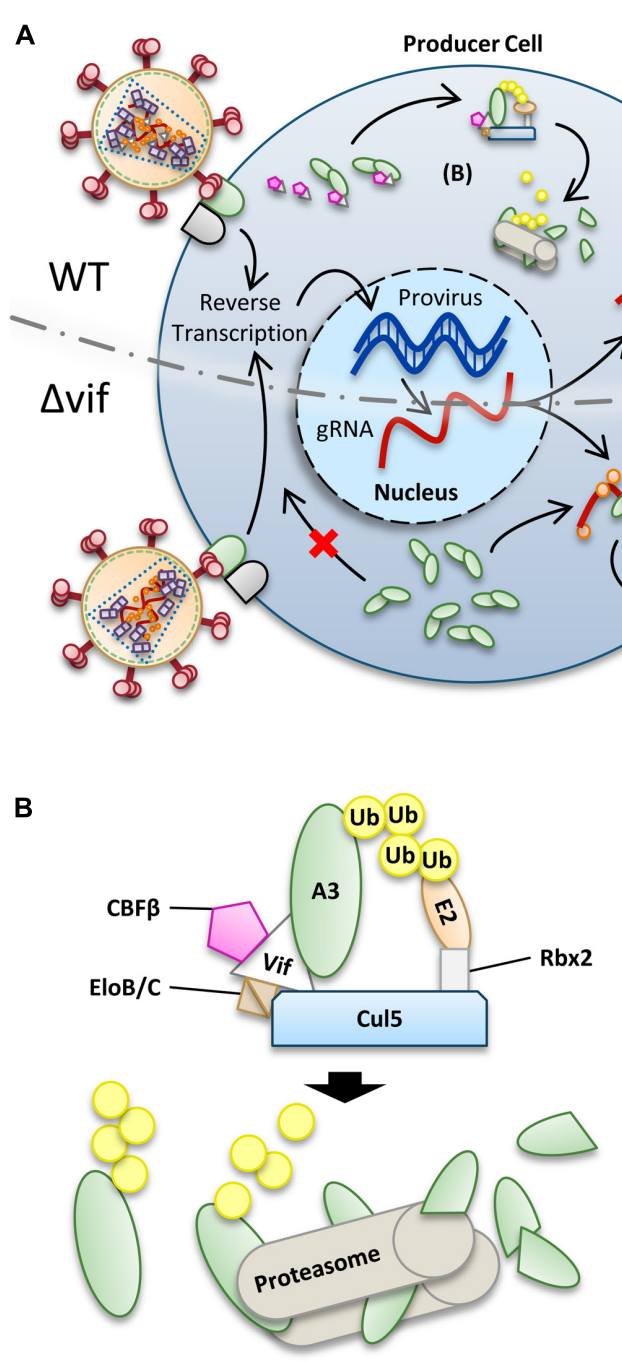

D

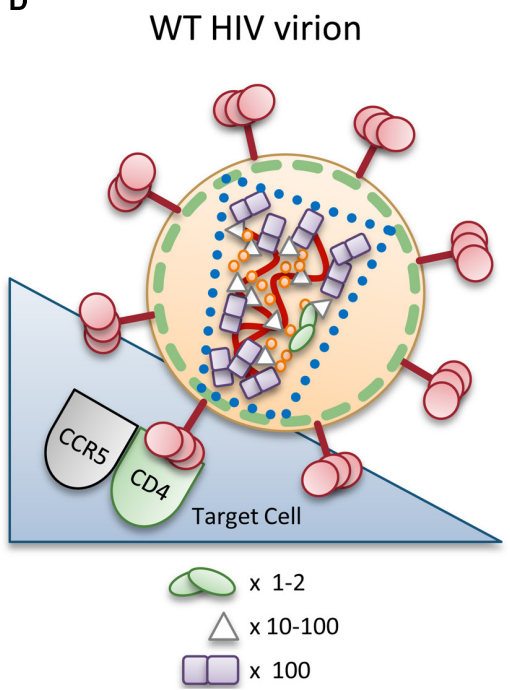

C

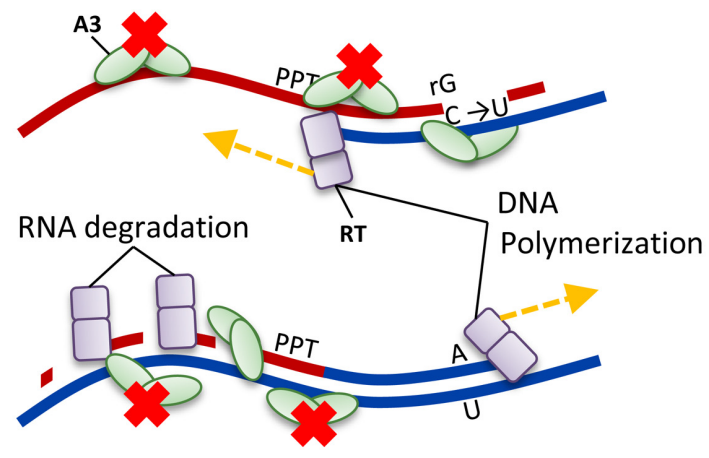

E

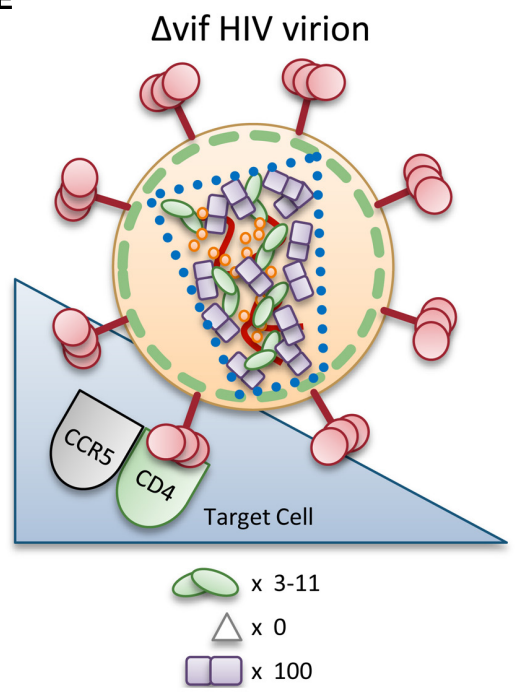




\section{FIGURE 2 | Continued}

Overview of HIV restriction by $\mathbf{A} 3$ enzymes. (A) Sketch depicting lifecycles of wild-type (WT) and $\Delta$ Vif HIV ( $\Delta$ Vif). Each virion enters a cell that expresses A3 enzymes. In the WT virus, Vif is expressed in the cell and recruits host cell CBF $\beta$ for stability and CRL5 E3 ubiquitin ligase complex composed of Elongin B/C (EloB/C), Cullin5 (Cul5) and Rbx2 (B). In this complex, Vif acts as the substrate receptor to induce degradation of $A 3$ enzymes. As a result, assembling virions do not encapsidate high levels of A3 enzymes and upon infection of a target cell the HIV lifecycle continues. The $\Delta$ Vif HIV encapsidates A3 enzymes through an RNA and Gag interaction. In the target cell the A3 enzymes within the capsid of HIV can deaminate cytosines to uracils in nascent single-stranded (-)DNA during reverse transcription $(\mathbf{C})$. These uracils induce $\mathrm{G} \rightarrow \mathrm{A}$ transition mutations upon synthesis of (+)DNA (C). The resulting hypermutated virus can be integrated into the host genome but is functionally inactivated. A3 enzymes in the target cell cannot enter the HIV capsid and are unable to restrict virus replication unless encapsidated into budding virions. (B) Detailed sketch of Vif-mediated polyubiquitination of $A 3 G$. Vif interacts with Elongin $C$ (EloC), which forms an obligate heterodimer with Elongin $B(E l o B)$, and Cul5. The transcription cofactor CBF $\beta$ stabilizes Vif. Cul5 binds to Rbx2and subsequently recruits an E2 ubiquitin conjugating enzyme. Vif is the substrate receptor that recruits $\mathrm{A} 3$ enzymes. The ${ }^{48} \mathrm{~K}$-linked ubiquitin chains result in proteasomal degradation of the $A 3$. (C) Sketch demonstrating the limited vulnerability of single-stranded (-)DNA to A3-mediated deamination that is imposed from the dynamics of reverse transcription. Reverse transcriptase is abbreviated as RT. HIV contains two polypurine tracts (PPT) that are used as primers for (+)DNA synthesis. In the figure, only one PPT is depicted. (D,E) Sketches depicting the stoichiometry of major virion components for a (D) WT and (E) $\triangle$ Vif HIV virion. Figures correspond to (D) and (E) in (A). (D) Low amounts of A3 may escape Vif-mediated degradation and become virion encapsidated (approximately one to two molecules of $A 3 G /$ virion). (E) A $\triangle$ Vif HIV cannot induce degradation of $A 3$ enzymes and that results in the encapsidation of $A 3$ enzymes through an interaction with RNA and Gag. Approximately 3-11 molecules of A3G can become virion encapsidated. (D,E) Stoichiometry values for virions were obtained from Camaur and Trono (1996), Fouchier et al. (1996), Coffin et al. (1997), Xu etal. (2007), Nowarski etal. (2008).

undergo successful integration with these hypermutations (Russell et al., 2009a), some preintegration complexes containing U may be degraded by host DNA repair mechanisms, although there is no consensus regarding the extent to which this occurs in cells (Kaiser and Emerman, 2006; Yang et al., 2007). In cell culture, it has been found that the mutated HIV proteins that may be produced from these proviral genomes can act as a source of HIV antigens due to their misfolding and processing through the proteasome, which can facilitate immune recognition of HIV (Casartelli et al., 2010).

Each ssDNA deaminase acts within a preferred di- or trinucleotide substrate motif. For A3G, this is $5^{\prime} \mathrm{CCC}$ or $5^{\prime} \mathrm{CC}$ (Yu et al., 2004, $3^{\prime}$-end $\mathrm{C}$ preferred for deamination). A3D, A3F, and A3H deaminate $5^{\prime} \mathrm{TTC}$ or $5^{\prime}$ TC motifs and $\mathrm{A} 3 \mathrm{D}$ can also deaminate 5'GC motifs in proviral DNA (Liddament et al., 2004; Dang et al., 2006; Ooms et al., 2013a). Although the majority of A3 actions are repressed by Vif in HIV-infected individuals (Figures 2A,B,D), clinical studies have found that individuals with an inherent ability to express a high level of A3G mRNA are less likely to become infected with HIV or progress from HIV to AIDS and that the presence of hypermutated proviral genomes correlates with high CD4+ T-cell counts (Jin et al., 2005; Pace etal., 2006; Biasin et al., 2007; Land et al., 2008; Vazquez-Perez et al., 2009). Other A3 enzymes have not been extensively examined in this regard (Albin and Harris, 2010; Ooms et al., 2013a). However, there is evidence of deaminations in HIV genomes recovered from infected individuals due to $\mathrm{C} / \mathrm{G} \longrightarrow \mathrm{T} / \mathrm{A}$ mutations in a sequence context that indicates deaminations by A3 enzymes other than A3G do occur (Pace etal., 2006; Land et al., 2008; Vazquez-Perez et al., 2009; Ooms etal., 2013a). For example, a study found that in HIV-infected individuals there is approximately an equal split between mutations occurring in the $5^{\prime} \mathrm{TC}$ and $5^{\prime} \mathrm{CC}$ contexts on (-)DNA (Ooms et al., 2013a). However, there is difficulty parsing out the effect of $\mathrm{A} 3 \mathrm{~F}, \mathrm{~A} 3 \mathrm{D}$, and $\mathrm{A} 3 \mathrm{H}$ based on their mutation patterns since they all recognize the minimal dinucleotide $5^{\prime} \mathrm{TC}$ and are more promiscuous than $\mathrm{A} 3 \mathrm{G}$ in regard to trinucleotide target site preference (Liddament et al., 2004; Dang et al., 2006; Hultquist etal., 2011). Nonetheless, it has become clear that despite some evidence that $\mathrm{A} 3 \mathrm{G}$ has more mutagenic potential than other A3 deaminases, it is not acting alone against HIV (Refsland et al., 2012; Chaipan et al., 2013; Ooms et al., 2013a). The HIV genomes mutated through A3 catalytic activity are also subject to the pressure of purifying selection. This selection pressure results in mutated and inactivated genomes being highest in integrated proviral DNA and lowest in circulating viral RNA (Russell et al., 2009a). Furthermore, integrated proviruses that are inactivated by stop codons in the gag gene may still be rescued by dual infection of a cell by HIV quasispecies and complementation of Gag function (Russell et al., 2009a). Recombination within virions by RT template switching can result in "reactivation" of inactivated viral genomes (Mulder et al., 2008; Russell et al., 2009a). As a result, A3-mediated mutagenesis is effective, but the complete inactivation of HIV in an infected individual is potentially a long-term process that is likely to require multiple rounds of exposure to viruses.

\section{A3G-MEDIATED RESTRICTION OF HIV \\ Deamination-dependent HIV restriction by A3G}

Since A3G was the first A3 enzyme discovered A3G has been the most widely studied for how it enacts its role as a restriction factor (over 700 publications in PubMed). There are two key steps that A3G must complete to be an efficient restriction factor. First, the enzyme must be available for binding RNA that will become virion encapsidated through an interaction with the NC portion of Gag (Alce and Popik, 2004; Cen et al., 2004; Douaisi et al., 2004; Svarovskaia et al., 2004; Burnett and Spearman, 2007; Khan et al., 2007; Bach et al., 2008; Bogerd and Cullen, 2008; Strebel and Khan, 2008; Wang etal., 2008). Second, it must have a mechanism to search the nascent HIV $(-)$ DNA, that is available for a finite period of time, for potential cytosines that it can deaminate (Chelico et al., 2006; Nowarski et al., 2008; Ara et al., 2014, Figure 2C).

A3G exists in cells as a high molecular mass that is bound to RNA and other proteins in stress granules and P-bodies (Chiu et al., 2006; Kozak et al., 2006; Wichroski et al., 2006; GalloisMontbrun et al., 2008). However, only newly synthesized A3G that has not associated with host RNAs in these cytoplasmic structures appears to bind the RNA that is also bound by HIV Gag and therefore encapsidated into virions (Soros et al., 2007). A3G requires oligomerization to bind these RNAs effectively in cells and become virion encapsidated (Wang et al., 2007; Bulliard et al., 2009; Huthoff et al., 2009), but in vitro oligomerization mutants of A3G can bind many RNAs with less than a threefold difference 
from wild-type (WT) A3G (Chelico et al., 2010; Feng and Chelico, 2011). The RNA binding and oligomerization of A3G is primarily mediated by the N-terminal domain (NTD) and the NTD is solely responsible for virion encapsidation of A3G (Hache etal., 2005; Navarro et al., 2005; Huthoff et al., 2009; Chelico et al., 2010, Figure 1). The NTD residues ${ }^{124} \mathrm{YYFW}^{127}$ on predicted loop 7 mediate the dimerization of A3G (Huthoff et al., 2009; Chelico et al., 2010, Figure 3A). A3G is primarily a dimer in solution and when A3G binds RNA or DNA it oligomerizes into tetramers and higher order structures through C-terminal domain (CTD) residues ${ }^{313}$ RIYDDQ ${ }^{318}$ on loop 7 (Chelico et al., 2008, 2010; Shlyakhtenko et al., 2011, Figure 3B). It is essential for $A 3 G$ to enter the inner capsid of the virion to restrict HIV. Within the capsid A3G can associate with the ribonucleoprotein complex and access the (-)DNA as it is synthesized. A3 enzymes that cannot encapsidate within the HIV capsid, e.g., $\mathrm{A} 3 \mathrm{~A}$ and $\mathrm{A} 3 \mathrm{C}$, are unable to restrict HIV replication in CD4+ T cells (Goila-Gaur et al., 2007; Hultquist et al., 2011). However, accessing the ribonucleoprotein complex does not guarantee the ability to restrict HIV. Since the $(-)$ DNA is only available for a finite period of time due to HIV containing two polypurine tracts (PPT) that are used to prime (+)DNA synthesis (Suspene et al., 2006; Hu etal., 2010), A3 enzymes require an efficient mechanism to search for cytosines (Figure 2C). Complicating the search is that the (-)DNA contains pieces of annealed RNA due to the endonuclease activity of the RT-associated $\mathrm{RNaseH}$ (Figure 2C). A3G binds RNA/DNA hybrids less well than ssDNA and encountering these obstacles on the substrate can induce A3G to dissociate from DNA (Iwatani et al., 2006; Chelico et al., 2008).

Unraveling the mechanism by which A3G locates and catalyzes its deamination motif is of pivotal importance for understanding the mechanistic basis of proviral hypermutation. The mechanism with which an A3 enzyme scans non-target DNA in search of its deamination motif is a determinant in its catalytic efficiency (Feng and Chelico, 2011; Ara et al., 2014). DNA scanning is described by the term processivity and is defined as the ability of an enzyme to catalyze multiple events in a single enzyme-DNA substrate encounter. Enzymes that do not use an energy source for movement on DNA use a mechanism termed facilitated diffusion to efficiently search DNA (Berg et al., 1981; von Hippel and Berg, 1989). This is a mechanism where the enzymes, subject to Brownian motion, move randomly on DNA. Since DNA-binding proteins are usually positively charged, the negative charge of the DNA facilitates the enzyme movement through electrostatic interactions (Berg et al., 1981; von Hippel and Berg, 1989). A3G is a positively charged enzyme (charge of +6.5 at $\mathrm{pH} 7$ ) and processively scans ssDNA by facilitated diffusion (Chelico et al., 2006; Nowarski et al., 2008, Figure 4). This mode is distinct from an enzyme that acts on DNA distributively, where only one catalytic event occurs before the enzyme disengages from the substrate (Chelico et al., 2009). Facilitated diffusion can involve a variety of movements such as 1-dimensional (D) sliding (Figure 4B) or 3-D movements such as hopping/jumping (Figure $4 \mathrm{C}$ ) or intersegmental transfer (Figure 4D, Halford and Marko, 2004). Hopping and jumping describe small microdissociations and reassociations with the same DNA strand without diffusion into the bulk solution (von Hippel and Berg, 1989, Figure 4C). Intersegemental transfer involves a two-step mechanism where an enzyme with two DNA-binding sites binds a second site before releasing the first site (von Hippel and Berg, 1989, Figure 4D). Facilitated diffusion works best when both 1- and 3-D movements are used to enable local scanning of a small segment of DNA by sliding $(<20 \mathrm{nt})$ and movement to distal regions to restart the local scanning process (Halford and Marko, 2004; Feng and Chelico, 2011). These distal movements do not cause the enzyme to leave the DNA and enter into the bulk solution because the charged surface of the DNA keeps the enzyme within the domain of the DNA (von Hippel and Berg, 1989, Figure 4C). Using different methods $\mathrm{A} 3 \mathrm{G}$ has been found to scan ssDNA by 1-D sliding motions and 3-D jumping motions (Chelico et al., 2006; Senavirathne et al., 2012; Shlyakhtenko et al., 2012). However, one study has found that $\mathrm{A} 3 \mathrm{G}$ moves by 3-D intersegmental transfers (Nowarski et al., 2008). The efficiency imparted by a 3-D movement in the specific case of $\mathrm{A} 3 \mathrm{G}$ during reverse transcription is that it provides a means of overcoming the DNA/RNA hybrid barrier (Nowarski et al., 2008; Feng and Chelico, 2011). Clusters of A3G-induced deaminations indicative of processive sliding movements have been found in integrated proviral genomes (Browne et al., 2009) and A3G mutants unable to undergo a local searching process by $1-D$ sliding, such as H186R and A3G with an ${ }^{195} \mathrm{NPM}^{197}$ insertion have decreased mutagenesis during in vitro reverse transcription or in HIV proviral genomes (Feng and Chelico, 2011; Ara et al., 2014). Furthermore, an A3G F126A/W127A mutant that cannot jump has decreased mutagenesis during in vitro reverse transcription (Feng and Chelico, 2011). These data demonstrate that neither movement alone can enable high levels of A3G-induced mutagenesis. Interestingly, the F126A/W127A mutant is monomeric, suggesting that the oligomeric state of A3G plays a role in efficient restriction of HIV not only by facilitating virion encapsidation but also by facilitating the DNA scanning process (Huthoff et al., 2009; Chelico et al., 2010; Feng and Chelico, 2011). The processivity determinants of $A 3 G$ reside on predicted loop 7 and helix 6 of the non-catalytic NTD (Feng and Chelico, 2011; Ara etal., 2014, Figure 3A). Thus, despite a lack of catalytic activity, the NTD contributes to A3G deamination activity by mediating the processive scanning mechanism (Feng and Chelico, 2011).

\section{Deamination-independent HIV restriction by A3G}

A3G primarily restricts HIV replication through its deamination activity. However, there have been numerous reports of an ability of $A 3 G$ to physically inhibit other processes of HIV such as RT polymerization (Iwatani et al., 2007; Bishop et al., 2008; Wang et al., 2012; Adolph et al., 2013; Belanger et al., 2013; Gillick et al., 2013), NC strand annealing (Guo et al., 2006, 2007), and proviral DNA integration (Luo et al., 2007; Belanger et al., 2013). These processes do not occur in isolation from deamination, nor do they restrict HIV better than deamination alone (Belanger et al., 2013; Gillick et al., 2013). We will focus on inhibition of RT polymerization since this is the most prevalent mechanism studied.

Early studies of A3G-mediated restriction of HIV proposed that transiently overexpressed WT A3G and deamination null mutants 
A

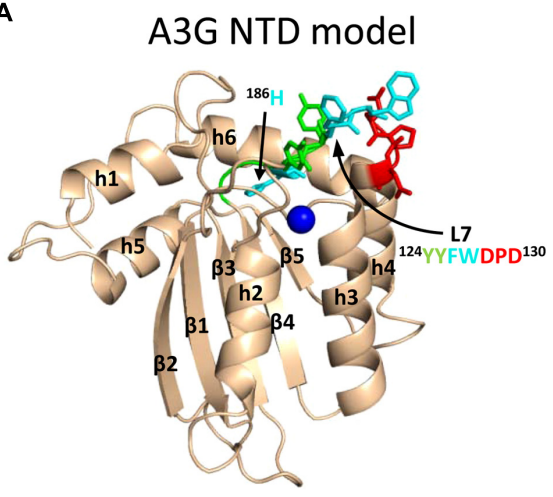

C

A3F NTD model

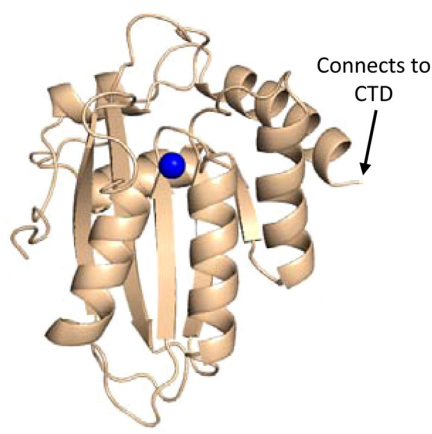

E

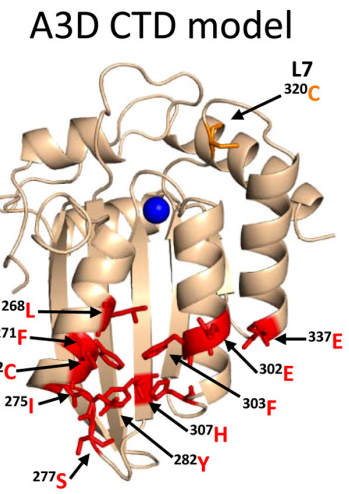

FIGURE 3 | Structures of $\mathbf{A} \mathbf{3}$ enzymes. A3 enzymes have a basic structure in each Z-type domain that is composed of a five-stranded $\beta$-sheet core surrounded by six $\alpha$-helices. Numerical assignments to $\beta$-strands and $\alpha$-helices are superimposed in (A). Zinc atoms are shown as blue spheres. (A) Model of the N-terminal domain (NTD) of A3G. Loop 7 (L7) of the A3G NTD is a central structure in its anti-HIV function. Highlighted on L7 are the residues important for interaction with Vif (red, ${ }^{128} \mathrm{DPD}^{130}$ ), oligomerization/virion encapsidation (green and cyan, ${ }^{124} \mathrm{YYFW}^{127}$ ), and jumping component of A3G processivity (cyan, ${ }^{126} \mathrm{FW}^{127}$ ). Helix 6 (h6) is adjacent to $L 7$ and contributes to the sliding component of A3G processivity, particularly ${ }^{186} \mathrm{H}$ (cyan). The model of the A3G NTD was obtained by using the automated SWISS-MODEL program using the homologous $A 3 G$ C-terminal domain structure (CTD, PDB: 3IOS). (B) The A3G CTD (PDB: 2KEM) is the catalytic domain of A3G. The A3G CTD has a discontinuous $\beta 2$ strand forming a loop-like bulge between the $\beta 2$ and $\beta 2^{\prime}$ strands. A3G L7 residues ${ }^{313}$ RIYDDQ ${ }^{318}$ (green) mediate tetramerization and determine the preferred deamination motif. (C) The model of the A3F NTD was obtained by using the automated SWISS-MODEL program using the homologous A3C structure (PDB: 3VM8). The end of h6 connects the NTD to the CTD and
B

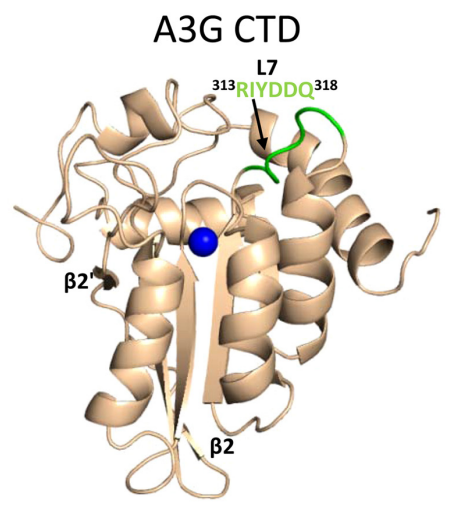

D

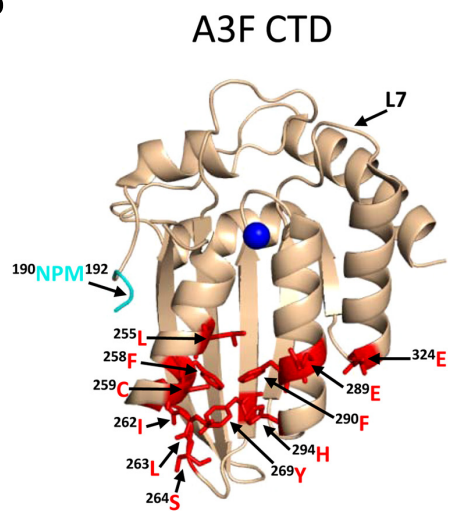

$\mathbf{F}$
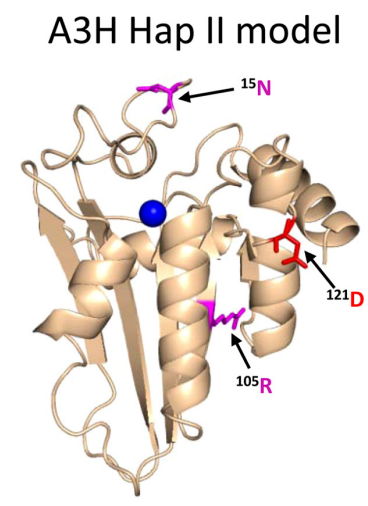

contains an ${ }^{190}$ NPM $^{192}$ motif. This NPM motif is found only in A3D and A3F. (D) The A3F CTD (PDB: $4 I O U$ ) is the catalytic domain of A3F and interacts with Vif. Residues that interact with Vif across Helix 2, 3, 4 , and $\beta$-strand 4 are shown in red. Also shown on this structure is the deamination motif specificity loop (L7) and the ${ }^{190}$ NPM $^{192}$ motif. The structure illustrates the kinked orientation introduced by the Pro in the ${ }^{190}$ NPM $^{192}$ motif, which blocks the sliding function of A3F. (E) The model of the CTD of A3D was obtained by using the SWISS-MODEL program using the homologous A3F structure (PDB: 4IOU). Residues that interact with Vif across Helix 2, 3, 4, and $\beta$-strand 4 are shown in red. The ${ }^{320} \mathrm{C}$ residue on $\mathrm{L} 7$ that influences $A 3 D$ activity is shown in orange. (F) Model of $\mathrm{A} 3 \mathrm{H}$ Hap II showing residues that interact with Vif and cause haplotype instability. In A3H Hap II, ${ }^{121} \mathrm{D}$ (red) on predicted h4 mediates an interaction with Vif. In A3H Hap I the R105G mutation induces protein instability (magenta). In $\mathrm{A} 3 \mathrm{H}$ Hap III and IV, the deletion of ${ }^{15} \mathrm{~N}$ induces protein instability (magenta). The model of the A3H Hap II was obtained by using the automated SWISS-MODEL program using the homologous APOBEC2 structure (PDB: 2NYT). Figures were made using PyMOL (The PyMOL Molecular Graphics System, Version 1.5.05, Shrödinger, LLC.). 


\section{A}

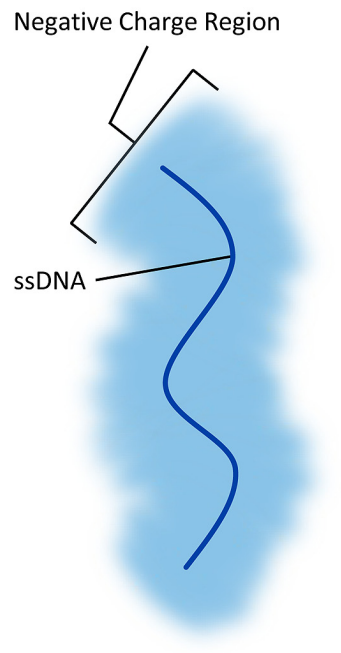

B

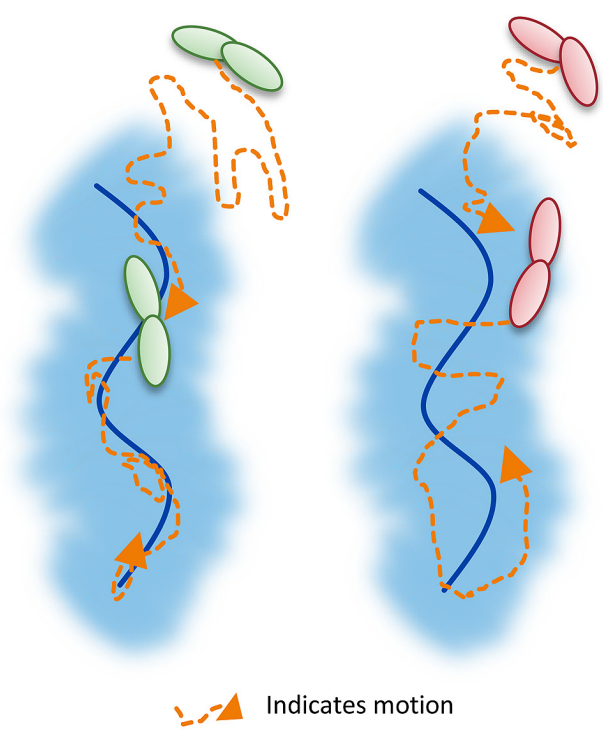

D
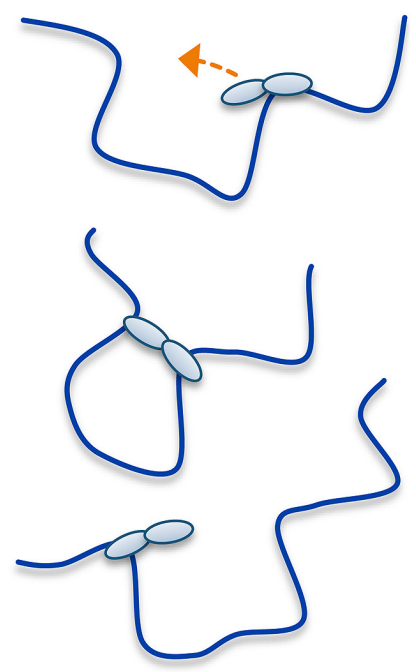

FIGURE 4 | Illustration of DNA scanning by facilitated diffusion.

(A) Sketch of DNA showing the negatively charged region of DNA important for facilitated diffusion of A3 enzymes. (B-D) Enzyme in sketches is shown as a dimer, although the oligomerization state may vary with different A3 enzymes. (B) Sketch depicting a 1-dimensional DNA scanning path by sliding. Dotted line indicates path of enzyme (orange). Sliding enables an in depth search of local areas of a substrate. (C) Sketch depicting a 3-dimensional DNA scanning path by jumping. Jumping enables larger translocations on DNA substrates, but lacks a local search process.
The microdissociations of the enzyme from the DNA that occur when the enzyme jumps does not leave the negatively charged domain of the DNA so the enzyme has a higher likelihood of reassociating with the same DNA substrate than diffusion into the bulk solution. (D) Sketch depicting a 3-dimensional DNA scanning path by intersegmental transfer. Intersegmental transfer enables larger translocations on DNA substrates, but lacks a local search process. An enzyme with two DNA-binding domains binds two regions of DNA simultaneously before dissociating from one region to move to another. of A3G could inhibit HIV proviral DNA formation (Mangeat et al., 2003; Newman etal., 2005). The initial mechanism proposed was that A3G binds the HIV genomic RNA which impedes RT (Iwatani et al., 2007). This has been confirmed in multiple reports using cellular and biochemical experiments (Adolph et al., 2013; Belanger et al., 2013; Chaurasiya et al., 2014). However, the physiological significance of these processes is difficult to reconcile since results from cell-based experiments using transiently expressed A3G cannot be replicated when A3G is stably expressed, suggesting that overexpression of $A 3 G$ induced experimental artifacts due to excessive packaging of $A 3 G$ in virions (Mbisa et al., 2007; Miyagi et al., 2007; Schumacher et al., 2008; Browne et al., 2009). Importantly, studies that used deamination null mutants of $\mathrm{A} 3 \mathrm{G}$ to show that deamination ability is required for restriction of HIV (Mbisa et al., 2007; Miyagi et al., 2007; Schumacher et al., 2008) should be considered in conjunction with data showing that the A3G E259Q catalytically inactive mutant is not a true proxy for A3G (Bishop etal., 2008; Adolph etal., 2013). A3G E259Q binds RNA less well than $\mathrm{A} 3 \mathrm{G}$ and this results in less inhibition of RT in vitro and in cells (Bishop et al., 2008; Adolph et al., 2013).

Nonetheless, it is clear that the ability of A3G to inhibit RT is highly dependent on $\mathrm{A} 3 \mathrm{G}$ concentration and the primer/template (Mbisa et al., 2007; Miyagi et al., 2007; Schumacher et al., 2008; Browne et al., 2009; Adolph etal., 2013). The initiation of DNA synthesis from an RNA primer on an RNA template is the least efficient type of polymerization activity of RT (Liu et al.,
2010). Accordingly, in vitro, low levels of A3G can best inhibit RT-mediated primer initiation at this step by competing for substrate (Iwatani et al., 2007; Adolph et al., 2013). In contrast, on a DNA primer and DNA template, A3G could at most inhibit in vitro RT-mediated initiation of synthesis by twofold under single turnover conditions and could not block RT from binding the primer/template, but merely lengthened the time RT required to find the free $3^{\prime} \mathrm{OH}$ (Adolph et al., 2013). These data are in agreement with a computational study that suggests an A3G-mediated deamination-independent mode of HIV restriction contributes $<1 \%$ of the restriction capability of A3G (Kobayashi et al., 2014). Although studies have shown that a peptide of A3G can interact with RT and inhibit RT-mediated DNA synthesis, it is unlikely that this mechanism is a physiological way to inhibit reverse transcription since in $\Delta$ Vif virions, only 3-11 A3G molecules are encapsidated whereas there are approximately 100 RT molecules (Coffin et al., 1997; Xu et al., 2007; Wang et al., 2012, Figure 2E). In the presence of Vif, there is only an estimated one to two molecules of A3G per virion (Nowarski et al., 2008, Figure 2D), emphasizing the importance of a deamination-dependent mechanism over a deamination-independent mechanism. A single molecule of $\mathrm{A} 3 \mathrm{G}$ could inactivate an HIV provirus through cytosine deamination whereas the deamination-independent mechanism is much more concentration dependent (Browne et al., 2009; Adolph et al., 2013). Single-molecule studies have brought forth the model that A3G oligomers can act as a road-block for HIV (Chaurasiya et al., 2014). Notably, existence of a deamination-independent 
mode of HIV inhibition has been observed ex vivo in primary cells (Gillick etal., 2013), but it requires further research as to the significance of this mode of inhibition during an HIV infection.

\section{A3F-MEDIATED RESTRICTION OF HIV \\ Deamination-dependent HIV restriction by A3F}

Approximately 2 years after the discovery of A3G, A3F was discovered to also exhibit restriction activity against HIV (Liddament et al., 2004; Wiegand et al., 2004; Zheng et al., 2004). Sequenced $\mathrm{HIV}$ proviral genomes were known to contain $\mathrm{G} / \mathrm{C} \longrightarrow \mathrm{A} / \mathrm{T}$ transition mutations in $5^{\prime} \mathrm{CC}$ and $5^{\prime} \mathrm{TC}$ contexts in the (-)DNA (Pathak and Temin, 1990; Li etal., 1991; Vartanian et al., 1991, 2002; Fitzgibbon et al., 1993) and A3F was found to contribute to transition mutations in the $5^{\prime} \mathrm{TC}$ context. Of these initial studies demonstrating A3F activity active against HIV (Bishop et al., 2004; Liddament et al., 2004; Wiegand et al., 2004; Zheng et al., 2004), all except one (Zennou and Bieniasz, 2006) found that A3F restriction activity was equivalent to $\mathrm{A} 3 \mathrm{G}$ restriction activity. It has since been shown that overexpression of A3 enzymes can result in excessive packaging into HIV virions and result in artifacts of HIV restriction (Miyagi et al., 2007; Xu et al., 2007; Schumacher et al., 2008). Yet even after 10 years of studying A3F, multiple groups still find different activities of A3F against HIV that cannot be attributable to overexpression, but perhaps different experimental systems and techniques (Miyagi et al., 2010; Mulder et al., 2010; Hultquist et al., 2011; Chaipan et al., 2013; Ara et al., 2014). However, A3F must exert a restriction pressure on HIV since Vif maintains an interaction interface with $\mathrm{A} 3 \mathrm{~F}$ that is distinct from $\mathrm{A} 3 \mathrm{G}$ in order to induce A3F degradation (Russell et al., 2009b). As with A3G, for A3F to effectively restrict HIV, it must be encapsidated with the ribonucleoprotein complex within the capsid (Wang et al., 2008) and effectively search for cytosines on the heterogeneous (-)DNA substrate (Ara et al., 2014).

A3F encapsidates into HIV virions through an interaction with RNA, but packages more efficiently than A3G into the core of HIV particles (Zennou and Bieniasz, 2006; Wang et al., 2008; Song et al., 2012). By resolving HIV capsids on a sucrose gradient to observe whether $\mathrm{A} 3 \mathrm{~F}$ and $\mathrm{A} 3 \mathrm{G}$ partition with the RNA and enzymes or the p24 capsid protein, Song et al. (2012) found that more A3F specifically associated with the ribonucleoprotein complex, in comparison to A3G. A3F binds nucleic acids with sevenfold higher affinity than A3G (Ara et al., 2014), which may enable it to package more specifically within the capsid (Song et al., 2012). Furthermore, A3F has been shown to bind doublestranded DNA with a higher affinity than A3G and maintain an association with the pre-integration complex of HIV as it enters the nucleus through its high-affinity nucleic acid binding (Mbisa et al., 2010; Burdick et al., 2013). Despite the quantity of A3F being at an equal or greater amount to A3G, A3F restricted HIV approximately fourfold less than $\mathrm{A} 3 \mathrm{G}$ in a single round of infection (Song etal., 2012). Although some reports show A3F can be less effective than A3G in restricting HIV (Miyagi et al., 2010; Mulder et al., 2010; Song et al., 2012; Chaipan et al., 2013; Ara et al., 2014), it cannot be concluded that it does not suppress HIV or impose selective pressure on HIV. Not only because of data showing A3F can effectively restrict HIV in spreading infections
(Hultquist et al., 2011; Refsland et al., 2012), but also because an HIV lab strain with tandem stop codons in Vif (from HIV NL43 ) will revert back to expressing a functional Vif in the presence of A3F (Albin etal., 2010a). This does not occur when A3G is used in the same type of forced evolution experiments (Hache et al., 2008, 2009). The HIV evolves to overcome A3G restriction, but does so by acquiring a $5^{\prime}$ UTR mutation to make HIV RNA transcription more efficient and altering the cell cycle through a Vpr mutation (Hache et al., 2008, 2009). Together these mutations result in more virus particles being produced. Presumably since A3G has less specific packaging in the capsid than $\mathrm{A} 3 \mathrm{~F}$, this strategy titrates out the ribonucleoprotein-packaged A3G enabling the HIV to escape high levels of mutagenesis. These data illustrate that A3G and A3F exert a distinct selective pressure on HIV due to distinct biochemical properties and that $\mathrm{A} 3$ packaging into virions is a necessary but insufficient step to ensure efficient HIV restriction (Ara et al., 2014).

To further understand why A3F-mediated restriction of HIV may be different than for A3G, Ara et al. (2014) undertook a biochemical study of A3F in comparison to A3G to identify biochemical differences between these enzymes that could account for differences in restriction efficiency. They found that in contrast to A3G, A3F used only 3-D jumping motions to scan ssDNA. This made the DNA scanning mechanism inefficient since A3F could translocate between many ssDNA regions and overcome intervening RNA/DNA hybrid regions, but lacked a local search mechanism to examine ssDNA regions for its $5^{\prime} \mathrm{TC}$ motif (Figure 4C). The A3F sliding movement is blocked by a ${ }^{190} \mathrm{NPM}^{192}$ motif in the connection domain between the NTD and CTD (Figures 3C,D) since mutagenesis of this motif to ${ }^{190} \mathrm{NGM}^{192}$ enabled A3F to slide (Ara et al., 2014). The Bohn et al.'s (2013) A3F CTD structure includes the ${ }^{190} \mathrm{NPM}^{192}$ sequence and shows that it is a kinked region of the loop structure (Figure 3D). Since an A3F ${ }^{190}{ }^{N G M}{ }^{192}$ mutant was able to slide, the data suggest that the rigid ${ }^{191} \mathrm{P}$ residue primarily blocks sliding. However, imparting sliding movement to A3F through the ${ }^{190} \mathrm{NGM}^{192}$ mutant did not increase A3F HIV restriction efficiency because the jumping movements of $\mathrm{A} 3 \mathrm{~F}$ differed from $\mathrm{A} 3 \mathrm{G}$ and were dominant over sliding which maintained an inefficient search of ssDNA (Ara etal., 2014). The differences in DNA scanning between $\mathrm{A} 3 \mathrm{~F}$ and $\mathrm{A} 3 \mathrm{G}$ were shown to be relevant to $\mathrm{HIV}$ restriction since A3F was fourfold less effective in restriction of HIV than A3G in a single-cycle infectivity assay (Ara et al., 2014). Of note, A3F was also shown to have a 100 -fold lower specific activity than A3G (Ara etal., 2014), but this was not thought to contribute to differences in restriction efficiency since studies with different $\mathrm{A} 3 \mathrm{G}$ and $\mathrm{A} 3 \mathrm{~F}$ mutants showed that mutagenesis efficiency correlated with the efficiency of the ssDNA scanning mechanism, not the specific activity. This is likely because RT polymerization and $\mathrm{RNaseH}$ activity limit the (-)DNA substrate available (Feng etal., 2013). The study by Ara etal. (2014) is in agreement with studies where A3F has not been as effective as A3G in restriction of HIV (Miyagi et al., 2010; Mulder etal., 2010; Chaipan etal., 2013), although A3F was found to be as restrictive to $\mathrm{HIV}$ replication as $\mathrm{A} 3 \mathrm{G}$ in other reports (Hultquist et al., 2011; Refsland et al., 2012). Despite A3F being considered in some reports to be less efficient than $\mathrm{A} 3 \mathrm{G}$ as an 
HIV restriction factor when considered side by side, this is far from the natural mechanism of these enzymes in which they act in concert (Refsland et al., 2012; Ooms et al., 2013a) and further studies examining how these enzymes work together are needed.

It is of note that Zennou and Bieniasz (2006) noticed that per mutation, A3F was less likely to inactivate HIV than A3G. This was later found to be because the $5^{\prime} \mathrm{CC}$ motif of A3G overlaps with the only Trp codon ( $5^{\prime}$ TGG/ACC) and results in a stop codon upon deamination of either cytosine in the motif (Yu et al., 2004). In contrast, A3F-induced mutations largely result in missense mutations which may or may not inactivate the encoded protein (Ara etal., 2014). The A3G 5'CC motif also overlaps with Gly codons and in the HIV prot mutations at these Gly results in more non-conservative mutations and gene inactivation than $\mathrm{A} 3 \mathrm{~F}$-induced missense mutations that primarily cause the conservative mutation of Glu to Gln (Ara et al., 2014). The determinant for motif specificity is loop 7 in the CTD (Langlois et al., 2005; Kohli et al., 2009; Carpenter et al., 2010; Rathore et al., 2013, Figures 3B,D). This loop can be grafted into different A3 enzymes to change site specificity (Kohli et al., 2009). However, the consequences of deamination mediated restriction can be independent from inducing amino acid changes. A3F and A3G may be able to block proviral integration through deoxycytidine deaminations that result in aberrant processing of the proviral DNA ends by HIV integrase and inhibition of plus-strand DNA transfer by reducing the efficiency of primer tRNA removal (Mbisa et al., 2007, 2010).

\section{Deamination-independent HIV restriction by A3F}

For many years, A3F was thought to have a stronger deaminationindependent mode of inhibiting HIV than A3G (Bishop et al., 2006; Holmes et al., 2007). Unlike A3G, the mechanism of deamination-independent "activity" was not extensively studied, but was presumed to be due to inhibition of RT polymerization. A computational study has found that A3G and A3F rely differentially on their deamination-independent modes of HIV restriction with $\mathrm{A} 3 \mathrm{G}$ only having the deamination-independent mode contributing to $<1 \%$ of its restriction activity whereas for A3F this value was approximately 30\% (Kobayashi et al., 2014). However, two studies using stably expressed A3F and A3F catalytic mutants C280S/C283A and E251Q demonstrated no inhibition of RT, suggesting that previous results were influenced by A3F overexpression artifacts (Miyagi et al., 2010; Albin et al., 2014). Another study showed that A3F can inhibit HIV integration by reducing $3^{\prime}$ processing of viral DNA at the U5 and U3 ends by integrase (Mbisa et al., 2010). Using a catalytic mutant of A3F (E251Q), the study found that inhibition of integration was decreased approximately twofold from that of WT A3F suggesting that catalytic activity is in part required to produce the aberrant U5 and U3 ends (Mbisa etal., 2010). Thus there appears to be consensus that despite the potentially inefficient mutagenic activity of $\mathrm{A} 3 \mathrm{~F}$ in some studies, the deamination activity of A3F is still dominant over the deamination-independent activity. Furthermore, if a deamination-independent mode of HIV inhibition functions in cells, it may be the inhibition of integration rather than reverse transcription (Mbisa et al., 2010).

\section{A3D-MEDIATED RESTRICTION OF HIV}

A3D was first characterized in 2006 to restrict HIV replication in single-cycle infectivity assays and to be suppressed by Vif, suggesting that it posed a restriction pressure on HIV (Dang et al., 2006). Further evidence of this was that HIV proviral genomes showed evidence of deaminations in the contexts of $5^{\prime} \mathrm{CC}, 5^{\prime} \mathrm{TC}$, and $5^{\prime} \mathrm{GC}$ (Dang etal., 2006). A3D was found to deaminate in the $5^{\prime} \mathrm{TC}$ and $5^{\prime} \mathrm{GC}$ contexts which were unique from $\mathrm{A} 3 \mathrm{G}$ and $\mathrm{A} 3 \mathrm{~F}$ that maintain less promiscuous deamination motif preferences (Dang et al., 2006). A3D also forms multimers through an RNA intermediate in cells with a similar profile as A3G (Li et al., 2014). In a clinical study of HIV-infected individuals, A3D was found to be upregulated in both Elite Controllers and in Non-Controllers but was down-regulated in response to successful anti-retroviral treatment, indicating that $\mathrm{A} 3 \mathrm{D}$ is part of a virological immune response to HIV (Abdel-Mohsen et al., 2013). However, the restrictive activity of A3D appears less than $\mathrm{A} 3 \mathrm{G}$ and $\mathrm{A} 3 \mathrm{~F}$ in single-cycle infectivity assays in cell lines (Dang etal., 2006, 2011; Hultquist et al., 2011) and spreading infections in primary human cells (Chaipan et al., 2013). Furthermore, $\mathrm{A} 3 \mathrm{D}$ represents the most divergent $\mathrm{A} 3$ enzyme in the lineage of chimpanzee to humans and the activity of $\mathrm{A} 3 \mathrm{D}$ has decreased from chimpanzees to humans (Duggal et al., 2011). Other chimpanzee and human A3 enzymes are more commonly found to have similar restriction potentials (Duggal et al., 2011). Chimpanzee A3D induces more hypermutation of HIV than human $\mathrm{A} 3 \mathrm{D}$, despite equivalent packaging into virions (Duggal et al., 2011). This was attributed to differences in loop 7 of the CTD (Dang etal., 2011; Duggal et al., 2011). One report found a single amino acid in the CTD loop 7, C320, that suppressed A3D antiviral activity (Dang et al., 2011, Figure 3E). If the C320 was replaced with a Tyr, as in A3F, the activity of A3D could be increased by more than 20-fold (Dang et al., 2011). In contrast, endogenous A3D from the T cell line CEM2n appears to have activity against $\mathrm{HIV}-1$ that is similar to $\mathrm{A} 3 \mathrm{~F}$ (Refsland et al., 2012). Using a series of A3 null backgrounds or A3 knockdowns, Refsland et al. (2012) found that the HIV-1 proviral hypermutation pattern at $5^{\prime} \mathrm{CC}$ and $5^{\prime} \mathrm{TC}$ sites was induced at comparable levels by the combined action of $\mathrm{A} 3 \mathrm{G}$ and $\mathrm{A} 3 \mathrm{~F}$ or A3G and A3D, suggesting a redundancy in the HIV-1 restriction mechanism.

\section{A3H-MEDIATED RESTRICTION OF HIV}

$\mathrm{A} 3 \mathrm{H}$ was originally identified as not being able to restrict HIV replication due to low steady-state protein levels in mammalian cells, despite normal mRNA expression (Dang et al., 2006; OhAinle et al., 2006). However, when A3H was recombinantly expressed in Escherichia coli it could mutate the E. coli genomic DNA (OhAinle et al., 2006). In later studies, it was realized that A3H exists as multiple haplotypes in the human population (Hap I-VII) with different stabilities in cells and HIV restriction capabilities (Table 1) and the original $\mathrm{A} 3 \mathrm{H}$ tested was an unstable form (Hap I, OhAinle et al., 2008; Harari et al., 2009). The unstable Hap I is the most prevalent form of $\mathrm{A} 3 \mathrm{H}$ in the population (Table 1), but is able to restrict HIV infection by approximately twofold when transiently overexpressed in cell lines (OhAinle et al., 2008; Harari et al., 2009; Li and Emerman, 2011; Wang et al., 2011a). Two amino acid 
Table 1 | Summary of A3H haplotype features.

\begin{tabular}{|c|c|c|c|c|c|c|c|c|c|}
\hline \multirow{2}{*}{$\begin{array}{l}\text { A3H } \\
\text { haplotypes }\end{array}$} & \multicolumn{5}{|c|}{ Polymorphic amino acid residues } & \multirow{2}{*}{$\begin{array}{l}\text { Antiviral activity } \\
\text { in cell culture }\end{array}$} & \multirow{2}{*}{$\begin{array}{l}\text { Protein } \\
\text { stability }\end{array}$} & \multirow{2}{*}{$\begin{array}{l}\text { Haplotype } \\
\text { frequency }\end{array}$} & \multirow[t]{2}{*}{ Reference } \\
\hline & $\Delta 15$ & 18 & 105 & 121 & 178 & & & & \\
\hline Hap II & $\mathrm{N}$ & $\mathrm{R}$ & $\mathrm{R}$ & $\mathrm{D}$ & $\mathrm{D}$ & Yes & Yes & $\begin{array}{l}0.061^{\mathrm{a}} \\
0.265^{\mathrm{b}}\end{array}$ & $\begin{array}{l}\text { OhAinle et al. (2008), Ooms etal. (2010), } \\
\text { Hultquist et al. (2011), Li and Emerman } \\
\text { (2011), Wang etal. (2011b), Zhen etal. } \\
\text { (2012), Ooms et al. (2013a) }\end{array}$ \\
\hline Hap V & $\mathrm{N}$ & $\mathrm{R}$ & $\mathrm{R}$ & $\mathrm{D}$ & $\mathrm{E}$ & Yes & Yes & $\begin{array}{l}0.202^{\mathrm{a}} \\
0.054^{\mathrm{b}}\end{array}$ & OhAinle et al. (2008), Wang et al. (2011b) \\
\hline Hap VI & $\Delta$ & $L$ & G & K & $\mathrm{D}$ & No & No & $\begin{array}{l}0.026^{a} \\
0.0004^{b}\end{array}$ & OhAinle et al. (2008), Wang et al. (2011 b) \\
\hline Hap VII & $\mathrm{N}$ & $\mathrm{R}$ & $\mathrm{R}$ & K & $E$ & Yes & Yes & $\begin{array}{l}0.009^{a} \\
\text { Not detected }\end{array}$ & OhAinle et al. (2008), Wang et al. (2011b) \\
\hline
\end{tabular}

aWang etal. (2011b).

b OhAinle etal. (2008).

polymorphisms, ${ }^{105} \mathrm{G}$ and $\Delta^{15} \mathrm{~N}$, can independently contribute to the instability of $\mathrm{A} 3 \mathrm{H}$ (Table 1). A3H Hap I is unstable due to a Gly at position 105 (OhAinle et al., 2008). An A3H Hap I G105R mutant (later identified as Haplotype VII, Table 1) renders the A3H stable in cells and imparts strong anti-HIV activity (OhAinle et al., 2008; Harari et al., 2009). Other unstable A3H haplotypes (III and IV) have the $\Delta^{15} \mathrm{~N}$ in combination with another polymorphism (OhAinle et al., 2008; Harari et al., 2009, Table 1). It is not known biochemically why these $\mathrm{A} 3 \mathrm{H}$ haplotypes are unstable, but comparative modeling of $\mathrm{A} 3 \mathrm{H}$ with the structure of a related family member APOBEC2 shows that amino acid 105 is in a $\beta$-strand within the central five-stranded $\beta$-sheet, suggesting that an R105G mutation could destabilize the core structure (Figure 3F). The $\Delta{ }^{15} \mathrm{~N}$ is predicted to be within a loop structure (Figure 3F) so it is difficult to predict the reason for the instability in this undefined region, but it is known from studies with A3F that deletions to a loop that connect the NTD and CTD cause protein instability (Ara et al., 2014), suggesting that the $\mathrm{A} 3 \mathrm{H}$ loop may need to be of a specific length for proper protein folding. Although different haplotypes (II, V, and VII) have been reported to exist in the population as stable forms that are able to restrict HIV (Table 1), in this review we focus only on $\mathrm{A} 3 \mathrm{H}$ Hap II (A3H Hap II), which has been the most highly studied. Notably, A3H Hap II has some variability in its restriction ability which is dependent on alternative mRNA spliced forms (Harari et al., 2009). A3H Hap II is primarily found in Africans/African Americans $(\sim 50 \%)$ and to a much lesser extent within other cultural populations (prevalence of approximately 0-8\%, OhAinle et al., 2008; Wang et al., 2011b). It has been proposed that $\mathrm{A} 3 \mathrm{H}$ evolved to become unstable due to a combination of the loss of an ancient pathogen and the ability of an ancestral $\mathrm{A} 3 \mathrm{H}$ to induce mutagenesis of genomic DNA (Jern and Coffin, 2008; OhAinle et al., 2008).

$\mathrm{A} 3 \mathrm{H}$ is the only $\mathrm{A} 3$ enzyme with highly diversified antiviral activities based on sequence polymorphisms ( $\mathrm{Li}$ and Emerman, 2011; Duggal etal., 2013) and appears to be in a category of its own in relation to other $\mathrm{A} 3$ enzymes regarding two other aspects. First, A3D, A3F, and A3G that also restrict HIV replication have two Z-type domains, whereas $\mathrm{A} 3 \mathrm{H}$ has only one Z-domain (LaRue etal., 2008, Figure 1). Phylogenic analyses have shown that the A3 Z-type domains have three distinct categories $(\mathrm{Z} 1, \mathrm{Z} 2$, and $\mathrm{Z3})$ and $\mathrm{A} 3 \mathrm{H}$ is the only $\mathrm{A} 3$ enzyme with an Z3 (LaRue etal., 2008, Figure 1). A3D and A3F have two Z2 domains and A3G has an Z1 (CTD) and Z2 (NTD) domain (LaRue et al., 2008, Figure 1). Second, A3H is the only single Ztype domain $\mathrm{A} 3$ (others are $\mathrm{A} 3 \mathrm{~A}$ and $\mathrm{A} 3 \mathrm{C}$ ) that forms oligomers and multimers. Structural and biochemical studies have found that $\mathrm{A} 3 \mathrm{~A}$ and $\mathrm{A} 3 \mathrm{C}$ are largely monomeric (>90\%) in solution and do not multimerize in cells through an RNA intermediate (Kitamura etal., 2012; Love etal., 2012; Byeon etal., 2013; Li et al., 
2014; Logue et al., 2014; Shlyakhtenko et al., 2014). In contrast, A3H Hap II was found to multimerize in cells (Li et al., 2014). The $\mathrm{A} 3 \mathrm{H}$ Hap II multimerization in cells was shown by fluorescence fluctuation spectroscopy and determined that multiple A3H Hap II molecules could closely associate on RNA, not that A3H Hap II oligomerized through a protein-protein interaction (Li et al., 2014). It remains to be determined if A3H Hap II can form a dimer in solution in the absence of RNA or DNA. A3G and $\mathrm{A} 3 \mathrm{~F}$ form oligomers in the absence of nucleic acid suggesting that $\mathrm{A} 3$ oligomerization ability facilitates the multimerization of A3 enzymes with RNA in cells (Chelico et al., 2008; Shlyakhtenko et al., 2011; Ara et al., 2014). It has been shown that similar to $\mathrm{A} 3 \mathrm{G}$ and $\mathrm{A} 3 \mathrm{~F}, \mathrm{~A} 3 \mathrm{H}$ Hap II interacts with cellular RNA and the NC portion of Gag to facilitate its encapsidation into HIV particles (Wang et al., 2011a; Zhen et al., 2012). Studies on A3H Hap II and Hap I have also shown that cytoplasmic localization correlates with restriction efficiency since mutation of $\mathrm{A} 3 \mathrm{H}$ Hap I to make it cytoplasmic (G105R) increases its restriction capacity despite other amino acid differences from $\mathrm{A} 3 \mathrm{H}$ Hap II (Harari et al., 2009; Li et al., 2010). Additionally, virion mislocalization of certain $\mathrm{A} 3 \mathrm{H}$ haplotypes may render them less active against HIV (Ooms etal., 2010). For example, despite nuclear localization of $\mathrm{A} 3 \mathrm{H}$ Hap I, it can be encapsidated into HIV particles, but through an association with the matrix and capsid region of Gag, which leads to its primary localization outside the capsid (Ooms et al., 2010). These data suggest that both cellular and virion localization play a role in restriction efficiency. There has been limited information in the literature on the biochemical properties of $\mathrm{A} 3 \mathrm{H}$ and how different haplotypes bind and scan ssDNA in search for deamination targets. A3H Hap II prefers to deaminate ssDNA at 5'TC sites, similar to $\mathrm{A} 3 \mathrm{~F}$ and $\mathrm{A} 3 \mathrm{D}$, and appears have a high mutagenic potential and ability to restrict HIV in both single-cycle and spreading infection experiments and in HIV-infected individuals (Harari et al., 2009; Hultquist et al., 2011; Wang et al., 2011a; Ooms et al., 2013a).

\section{RESTRICTION OF HIV BY COORDINATELY EXPRESSED A3 ENZYMES}

Vif-deficient HIV showed replication defects when produced from cell lines such as CEM and H9, resulting in their classification as non-permissive cell lines (Gabuzda et al., 1992; Blanc et al., 1993; Sakai et al., 1993; von Schwedler et al., 1993; Madani and Kabat, 1998; Simon etal., 1998). After many years of investigating the function of Vif and trying to understand the dichotomous phenomenon of permissive and non-permissive cell lines for $\Delta \mathrm{Vif}$ HIV, Sheehy et al. (2002) found that the non-permissive CEM cell line expressed A3G. Thereafter, many groups discovered that Vif was required to induce degradation of $\mathrm{A} 3 \mathrm{G}$ to enable HIV replication (Sheehy et al., 2002, 2003; Conticello et al., 2003; Kao et al., 2003; Mariani et al., 2003; Marin et al., 2003; Stopak et al., 2003; Yu et al., 2003). Later, it was realized by analyzing the mRNA expression levels of A3s using quantitative PCR in permissive (CEM-SS, SupT1) and non-permissive (CEM and H9) T cell lines that the classical non-permissive CEM T cell line expressed not only A3G but also A3F and A3D, albeit with lower mRNA levels (Refsland et al., 2010). It is interesting to speculate whether more data would be available on the inhibition of HIV by the combined action of multiple A3s if they were discovered at the same time as $\mathrm{A} 3 \mathrm{G}$.

In primary $\mathrm{CD} 4+\mathrm{T}$ cells $\mathrm{A} 3$ enzymes relevant to HIV restriction are expressed and further induced by mitogens, rather than interferon, indicative of their function in restricting retrotransposons (Koning et al., 2009; Refsland et al., 2010). In contrast, in macrophages, monocytes and dendritic cells expression of select A3 enzymes is induced by interferon (Koning et al., 2009; Refsland et al., 2010). Although A3 enzymes are not individually expressed in cells as in many laboratory experiments (Refsland et al., 2010, 2012), there is an advantage of individual expression of each A3. Individual expression of A3 enzymes enables mechanistic information to be learned about enzyme function and mutational footprints established. However, A3s with perhaps a lesser restriction efficiency would not be expressed alone during an HIV infection suggesting that it may not matter per se which enzyme is most effective since they may each contribute cooperatively to HIV restriction. Gillick et al. (2013) found that in primary human CD4+ $\mathrm{T}$ cells the majority of proviral mutations were in a sequence context that indicated A3G-induced mutations are dominant $\left(5^{\prime} \mathrm{CC}\right)$, but $\mathrm{A} 3 \mathrm{~F}$ - and $\mathrm{A} 3 \mathrm{D}$-induced mutations $\left(5^{\prime} \mathrm{TC}\right.$ context) were evident at ninefold less frequency than the $5^{\prime} \mathrm{CC}$ context in $\triangle$ Vif HIV. This is in contrast to a study by Ooms et al. (2013a) that used peripheral blood mononuclear cells to examine the hypermutation of HIV in the absence or presence of $\mathrm{A} 3 \mathrm{H}$ Hap II. In the absence of A3H Hap II, it was found using a deep sequencing approach that there was approximately an equal number of mutations originating in $5^{\prime} \mathrm{CC}$ and $5^{\prime} \mathrm{TC}$ contexts, suggesting that $\mathrm{A} 3 \mathrm{~F}$ and $\mathrm{A} 3 \mathrm{D}$ cooperate to induce an equivalent number of mutations to A3G (Ooms et al., 2013a), in agreement with results from a CEM2n T cell line (Refsland et al., 2012). In the presence of Vif that could induce degradation of all A3s except A3H Hap II, there was a large number of mutations in the $5^{\prime} \mathrm{TC}$ context demonstrating that $\mathrm{A} 3 \mathrm{H}$ when present in a stable form is highly active against HIV (Ooms et al., 2013a).

Although the use of spreading infections in primary cells or $\mathrm{T}$ cell lines supports the idea that A3s cooperate, there still may be a question of whether they induce HIV evolution. It has been proposed that if there is an insufficient amount of A3-induced hypermutation this may benefit HIV and contribute to sequence variation by induction of sublethal levels of mutagenesis which results in HIV evolution (Mulder et al., 2008; Kim et al., 2010; Sadler et al., 2010; Monajemi et al., 2014). There is evidence that A3G and A3F hotspots are enriched in immunogenic CTL epitopes and that HIV may utilize A3s to induce immune escape (Monajemi et al., 2014). In addition, A3G may be able to induce resistance to the RT inhibitor lamivudine (3TC) because its deamination motif overlaps with a codon for Met and results in an M146I mutation in the pol gene (Mulder et al., 2008; Kim et al., 2010). However, the frequency of this mutation being induced by A3G versus RT activity has been questioned (Jern et al., 2009). It is also not known if $\mathrm{A} 3 \mathrm{~F} / \mathrm{A} 3 \mathrm{D} / \mathrm{A} 3 \mathrm{H}$ Hap II induce this evolution any more than $A 3 G$, due to differences in inactivation potential derived from their sequence specificities (Yu et al., 2004; Zennou and Bieniasz, 2006; Love et al., 2012; Ara et al., 2014) and if this impacts disease progression in infected individuals. On the other hand, Vif has been shown to adapt within HIV-infected individuals and be less 
effective in inducing A3 degradation (Simon et al., 2005; Fourati et al., 2010). It is thought that HIV can utilize Vif as a mutational rheostat in times of viral stress by allowing low amounts of A3s into viral particles to induce sublethal mutagenesis (Simon et al., 2005; Fourati et al., 2010). These types of studies have raised the idea that perhaps inducing hypomutation or shutting off A3 enzymes may benefit HIV-infected individuals (Harris, 2008; Hultquist and Harris, 2009).

\section{HIV Vif}

\section{GENERAL PROPERTIES}

The main function of Vif remained elusive at the beginning of HIV research, except for the finding that Vif made some cell lines permissive for producing HIV particles capable of undergoing another round of infection (Fisher et al., 1987; Strebel et al., 1987). Non-permissive cells allowed a $\Delta$ Vif HIV to produce virus particles, but they were rendered non-infectious upon infection of fresh cells. Two laboratories discovered that Vif repressed a host factor (Madani and Kabat, 1998; Simon et al., 1998). It was later identified by subtractive hybridization that A3G (originally called CEM15) was the host factor that was highly packaged into virions in the absence of Vif and blocked infection in the next target cell (Sheehy et al., 2002, Figure 2A). Although this is clearly a primary role for HIV infectivity, Vif was also shown to influence HIV particle morphology and this may relate to its potential role as a nucleic acid chaperone (von Schwedler et al., 1993; Hoglund et al., 1994; Henriet et al., 2007; Batisse et al., 2012).

\section{Vif AS AN E3 UBIQUITIN LIGASE SUBSTRATE RECEPTOR}

In 2012 it was discovered that Vif interacts with the host transcription cofactor CBF $\beta$ for stability in cells (Jager et al., 2012; Zhang et al., 2012). The interaction is mediated through Vif amino acids ${ }^{84} \mathrm{GxSIEW}^{89}$ and ${ }^{102}$ LADQLI $^{107}$ (Matsui et al., 2014b; Wang et al., 2014, Figures 5A,B). The Vif/CBF $\beta$ complex is also required for in vitro stability of Vif and enables recombinant expression of Vif in a largely soluble form in E. coli that can be purified for biochemical studies (Zhou et al., 2012). In contrast, Vif alone expressed in E. coli accumulates in inclusion bodies and must be purified under denaturing conditions (Yang et al., 1996). To suppress A3 action Vif interacts directly with A3 enzymes and mimics the human protein suppressor of cytokine signaling-2 (SOCS2) to become the substrate recognition subunit of a Cullin RING ligase-5 (CRL5) E3 ligase complex (Figure 2B).

Vif interacts with host proteins Elongin $\mathrm{C}$, which forms an obligate heterodimer with Elongin B (EloB/C) and Cullin 5 (Cul5, Marin et al., 2003; Yu et al., 2003, 2004; Mehle et al., 2004; Luo et al., 2005; Xiao et al., 2007; Stanley et al., 2008; Bergeron et al., 2010). The interaction of Vif with EloB/C increases the stability of Vif in cells and in vitro and promotes recruitment of CBF $\beta$ (Wang et al., 2013). The interaction of Vif with EloC is mediated through an SLQ motif in Vif termed the Elongin B/C (BC) box (Yu et al., 2003, 2004, Figures 5A,B, ${ }^{144}$ SLQYLA $^{149}$ ), similar to human SOCS proteins (Kamura et al., 1998; Iwai et al., 1999). Distinct from human proteins is that Vif does not have the highly conserved Cys in the BC box and instead has a ${ }^{149} \mathrm{~A}$ (Kamura et al., 1998, 2004, Figures 5A,B). The data with Vif suggest that it is the short side chain of the amino acid at position 149 rather than the Cys that is required for the interaction with EloC (Yu et al., 2004; Stanley et al., 2008). Vif also does not contain a canonical Cul5 box (Luo et al., 2005; Xiao et al., 2007). In search of the conserved Cys in the BC box, two other Cys $\left({ }^{114} \mathrm{C},{ }^{133} \mathrm{C}\right)$ were identified in Vif upstream of the $\mathrm{BC}$ box and were found to be involved in binding with Cul5 (Mehle et al., 2004; Yu et al., 2004). These Cys were found to be part of a novel Zinc binding $\mathrm{HCCH}$ motif (Figures 5A,B, ${ }^{108} \mathrm{Hx}_{2} \mathrm{YFxCFx}_{4} \mathrm{IRx}_{2} \mathrm{LxGx}_{6} \mathrm{CxY}_{3} \mathrm{H}^{139}$ ). The Zinc coordination in the $\mathrm{HCCH}$ was predicted to stabilize a small domain of Vif and indirectly support Cul5 binding (Luo et al., 2005). The primary Vif amino acids that contact Cul5 are at positions 120-121 and 124 in a helix that is adjacent to the HCCH residues (Xiao et al., 2006; Guo et al., 2014, Figures 5A,B, ${ }^{120} \mathrm{IRxxL}^{124}$ ). The Vif/CBF $/$ /EloB/C heterotetramer undergoes a conformational change that promotes binding to Cul5, suggesting that there is a prescribed order in the assembly of the E3 CRL5 ligase complex (Fribourgh et al., 2014). Accordingly, Cul5 binds less well to EloB/C in the absence of Vif/CBFß (Guo et al., 2014).

A recent structural study of Vif bound to $\mathrm{CBF} \beta / \mathrm{EloB} / \mathrm{C} / \mathrm{Cul} 5$ shows that Vif has an overall elongated cone structure and contains two domains with a Zinc binding domain in the center of the two domains (Guo et al., 2014, Figure 5C). CBF $\beta$ binds the $\mathrm{N}$-terminal $\alpha / \beta$-domain and EloC and Cul5 bind the C-terminal $\alpha$-domain of Vif (Guo et al., 2014, Figure 5C). Both EloC and Cul5 interact with Vif through hydrophobic interfaces on distinct $\alpha$-helices (Xiao et al., 2006; Guo et al., 2014). The crystal structure also emphasizes the stability that $\mathrm{CBF} \beta$ imparts to Vif since they have a total interaction surface area of $4797 \AA^{2}$ and form an antiparallel $\beta$-sheet with a $\beta$-strand from each protein (Guo et al., 2014). The side of CBF $\beta$ that is bound by Vif is the same side that the human CBF $\beta$ binding partner, the RUNX1 transcription factor binds to suggesting a mutually exclusive binding (Kim et al., 2013; Guo et al., 2014), although other reports show CBF $\beta$ can bind Vif and RUNX1 on genetically distinct surfaces (Hultquist et al., 2012; Zhang et al., 2012; Du et al., 2013a). Functionally, Vif appears to exclude CBF $\beta$ from binding RUNX1 because expression of Vif can alter the RUNX1-dependent transcriptional profile of cells and suggests that Vif may have multimodal effects in HIV-infected cells (Kim et al., 2013).

$\mathrm{CBF} \beta$ interacts with a hydrophobic region of the Vif $\alpha / \beta$ domain, but the rest of the exposed $\alpha / \beta$-domain surface is highly positively charged and is thought to mediate electrostatic interactions with A3 enzymes (Aydin et al., 2014; Guo et al., 2014). To target A3s for proteasomal degradation, Cul5 interacts with RING finger protein 2 (Rbx2, Jager et al., 2012) and this results in the assembly of a hexameric complex (Figure 2B). Furthermore, an E2 ubiquitin conjugating enzyme interacts with the hexamer through $\mathrm{Rbx} 2$ and causes ${ }^{48} \mathrm{~K}$-linked polyubiquitination of the A3 enzyme, on multiple lysine residues signaling it for degradation through the proteasome pathway (Figure 2B). Current data for A3G and A3F suggest that the Lys residues that become conjugated to ubiquitin are random (Albin et al., 2013).

\section{Vif amino acids that interact with A3s}

Alanine scanning mutagenesis of Vif or comparison of different Vif variants from HIV subtypes has enabled the identification of 
A

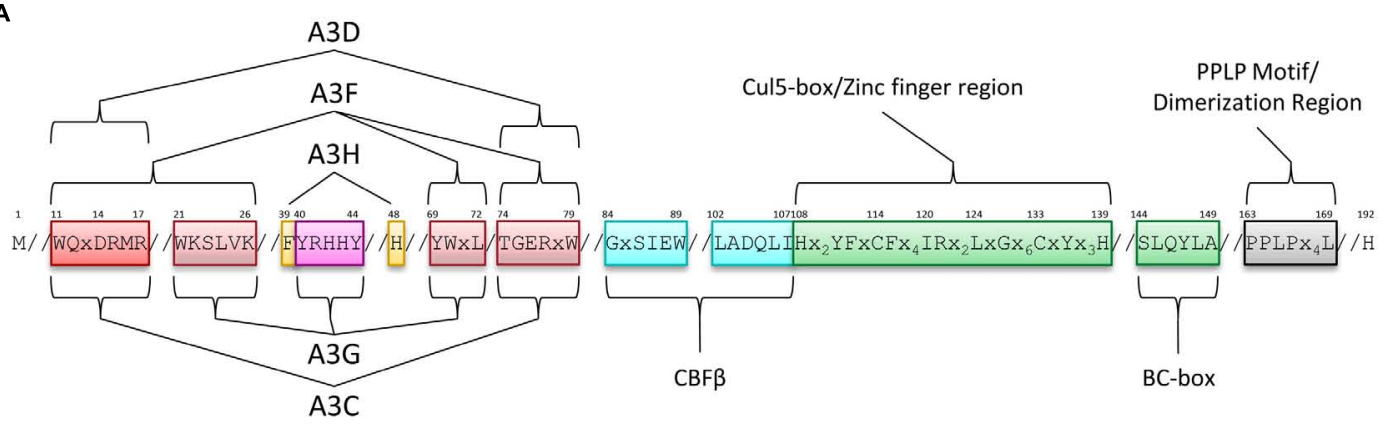

B

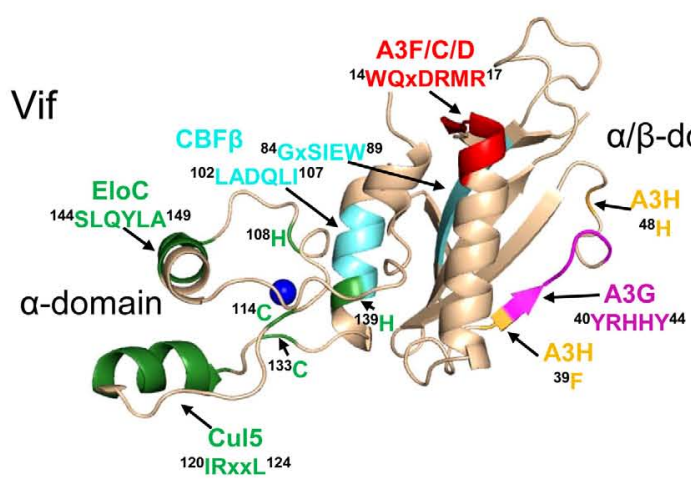

C

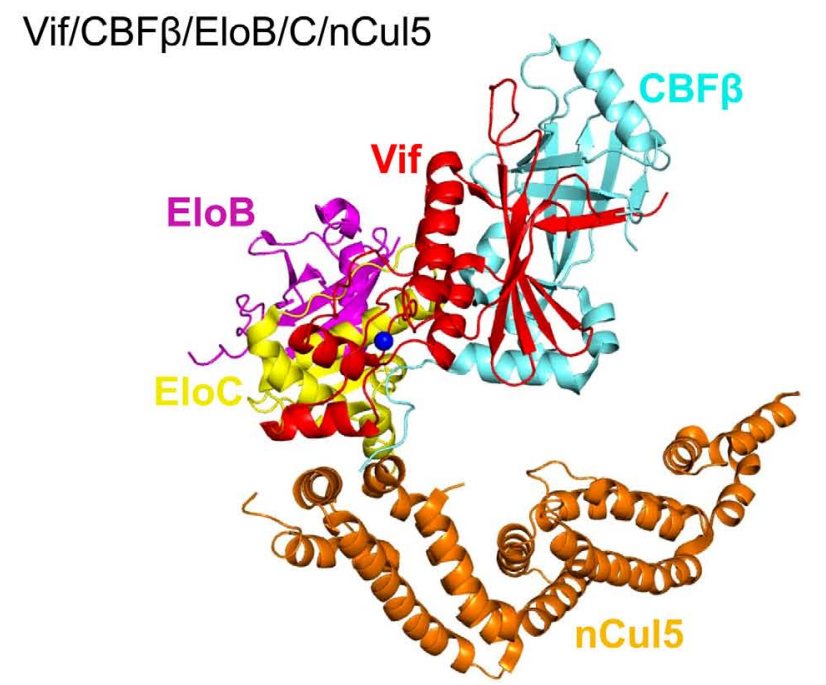

FIGURE 5 | Structure of Vif and host interacting partners. (A) Domain organization of Vif. Vif uses specific motifs to interact with A3G (magenta, ${ }^{40} \mathrm{YRHHY}^{44}$ ), A3F/A3C/A3D (red, ${ }^{11}$ WQXDRMR ${ }^{17}$ and ${ }^{74} \mathrm{TGER} \times \mathrm{W}^{79}$ ), and $\mathrm{A} 3 \mathrm{H}$ (orange, ${ }^{39} \mathrm{~F}$ and ${ }^{48} \mathrm{H}$ ). In conjunction with these specific motifs, there are shared interaction motifs for $\mathrm{A} 3 \mathrm{~F}$ and $\mathrm{A} 3 \mathrm{G}$ with Vif (pink, ${ }^{21}$ WKSLVK ${ }^{26}$ and ${ }^{69} \mathrm{YWXL}{ }^{72}$ ). $\mathrm{CBF} \beta$ interacts with Vif through two adjacent motifs (cyan, ${ }^{84} \mathrm{GXSIEW}^{89}$ and ${ }^{102} \mathrm{LADQLI}^{107}$ ). The Zinc finger region (green, amino acids 108-139) coordinates the Zinc through an ${ }^{108} \mathrm{H}^{114} \mathrm{C}^{133} \mathrm{C}^{139} \mathrm{H}$ motif and stabilizes the Vif structure, which indirectly enables an interaction with Cullin 5 (Cul5). Direct interaction of Vif with Cul5 is through amino acids ${ }^{120} I_{R \times x L}{ }^{124}$. The $B C$ box mediates an interaction with Elongin $C$ (green ${ }^{144}$ SLOYLA ${ }^{149}$ ). Vif oligomerizes through a PPLP motif (gray,

${ }^{163} \mathrm{PPLPx}_{4} \mathrm{~L}^{169}$ ). Slanted lines are used to indicate intervening amino acids between the domains. (B) The crystal structure of Vif (PDB: 4N9F) shows that it has two domains on either side of a bound Zinc (blue). The $N$-terminal $\alpha / \beta$-domain consists of a five stranded $\beta$-sheet, a discontinuous $\beta$-strand and three $\alpha$-helices. The $\alpha / \beta$-domain contains the binding interface for CBF $\beta$ (cyan, ${ }^{102}$ LADQLI 107, ${ }^{84} \mathrm{GxSIEW}{ }^{89}$ ) and A3 enzymes. The

${ }^{11}$ WQXDRMR ${ }^{17}$ motif (red) is used to interact with $A 3 F, A 3 C$, and $A 3 D$, the ${ }^{40} \mathrm{YRHHY}^{44}$ motif (magenta) is used to interact with $\mathrm{A} 3 \mathrm{G}$, and residues ${ }^{39} \mathrm{~F}$ and ${ }^{48} \mathrm{H}$ (orange) are used to interact with $\mathrm{A} 3 \mathrm{H}$. The $\alpha$-domain contains two alpha helices that mediate two separate interactions with EloC (green, ${ }^{144}$ SLOYLA ${ }^{149}$ ) and Cul5 (green, ${ }^{120} I R \times x L^{124}$ ). (C) Structure HIV Vif (red) in complex with $\mathrm{CBF} \beta$ (cyan), Elongin $\mathrm{C}$ (EloC, yellow), and the $\mathrm{N}$-terminal domain of Cullin 5 (nCul5, amino acids 12-386, orange, PDB: 4N9F). Elongin $\mathrm{B}$ (EloB, magenta) dimerizes with EloC. Figures were made using PyMOL (The PyMOL Molecular Graphics System, Version 1.5.05, Shrödinger, LLC.). 
three distinct regions of Vif that interact with A3G, A3F/A3D/A3C, or A3H (Huthoff and Malim, 2007; Russell et al., 2009b; Binka etal., 2012, Figure 5A). Vif interacts with A3G through two positively charged regions on Vif, ${ }^{21} \mathrm{WxSLVK}^{26}$ and ${ }^{40} \mathrm{YRHHY}^{44}$ (Mehle etal., 2007; Russell and Pathak, 2007; Yamashita et al., 2008; Chen etal., 2009; Dang et al., 2009, Figures 5A,B). Similarly, various domains in Vif have been identified to interact with A3F, specifically ${ }^{11} \mathrm{WQxDRMR}^{17}$ and ${ }^{74} \mathrm{TGERxW}^{79}$ (Tian et al., 2006; Russell and Pathak, 2007; He et al., 2008; Yamashita et al., 2008, Figures $\mathbf{5 A}, \mathbf{B}$ ). In addition, the ${ }^{69} \mathrm{YWxL}^{72}$ motif is a region of Vif that interacts with both $\mathrm{A} 3 \mathrm{G}$ and $\mathrm{A} 3 \mathrm{~F}$ (He et al., 2008; Pery etal., 2009, Figure 5A). However, for both A3G and A3F mutation of the ${ }^{40} \mathrm{YRHHY}^{44}$ and ${ }^{14} \mathrm{DRMR}^{17}$ motifs to all alanines are necessary and sufficient to block Vif-induced A3G and A3F degradation, respectively, suggesting the other domains provide a secondary stabilizing interaction (Russell and Pathak, 2007). Of note, A3C and A3D share a common binding site on Vif as A3F with ${ }^{14} \mathrm{DRMR}^{17}$ shown to be of importance (Pery et al., 2009; Kitamura et al., 2011, 2012, Figures 5A,B). Vif interacts with $\mathrm{A} 3 \mathrm{H}$ through another unique site that involves amino acid ${ }^{39} \mathrm{~F}$ and ${ }^{48} \mathrm{H}$ (Binka et al., 2012; Ooms et al., 2013b, Figures 5A,B).

The Vif amino acids that interact with $\mathrm{A} 3 \mathrm{H}$ are not highly conserved among HIV subtypes, in contrast to the motifs of Vif that interact with A3G and A3F/A3D. It has been suggested that since HIV rarely encounters a host with an active $\mathrm{A} 3 \mathrm{H}$ allele, there has been evolutionary drift of Vif to not maintain an interaction site with $\mathrm{A} 3 \mathrm{H}$ (Ooms etal., 2013a). As a result, A3H is differentially sensitive to Vif variants. For example, A3H Hap II is not sensitive to HIV NL4-3 Vif $\left({ }^{39} \mathrm{~F},{ }^{48} \mathrm{~N}\right)$, but is sensitive to HIV LAI Vif $\left({ }^{39} \mathrm{~F},{ }^{48} \mathrm{H}\right.$, Ooms etal., 2013b). The inability of some Vif variants to induce degradation of A3H Hap II enabled Ooms et al. (2013a) to test whether A3H could act as an infection barrier to HIV. Ooms et al. (2013a) found that Vif will adapt in infected individuals to induce degradation of A3H Hap II (Li et al., 2010). Importantly, this evolution of Vif affects only A3H and Vif maintains the ability to induce degradation of $A 3 G$ and A3F (Ooms et al., 2013a), confirming that Vif indeed uses three distinct interfaces to interact with A3 enzymes and supports the idea that multiple $\mathrm{A} 3$ enzymes coordinately exert a restriction pressure on HIV. Importantly, treatment naïve HIV-infected individuals at the early or primary infection stage that had at least one active $\mathrm{A} 3 \mathrm{H}$ allele (Hap II) had higher levels of mutations in proviral genomes in a $5^{\prime} \mathrm{TC}$ context, lower viral loads and higher CD4+ T cell counts (Ooms et al., 2013a). Gourraud et al. (2011) similarly reported that early stage, untreated HIV-infected individuals that were homozygous for a stable $\mathrm{A} 3 \mathrm{H}$ allele demonstrated lower HIV RNA over time, but this did not correlate with increased hypermutation of HIV proviral genomes. This difference in mutational load between these reports is likely due to the different sequencing strategies used between the two studies (Gourraud etal., 2011; Ooms et al., 2013a). These data are similar to clinical data obtained with A3G and A3F that demonstrate in a number of cohorts (but not all), A3G or A3F mRNA expression or hypermutation levels correlate with high CD4+ $\mathrm{T}$ cell counts and low viremia (reviewed in Albin and Harris, 2010).

\section{Vif-A3G interaction}

The Vif-A3G interaction was the first Vif-A3 interaction to be studied and it established that Vif inhibits the antiviral activity of A3 enzymes in a species-specific manner (Bogerd et al., 2004; Mangeat et al., 2004; Schrofelbauer et al., 2004; Xu et al., 2004; Etienne et al., 2013; Letko et al., 2013). This means that HIV Vif cannot neutralize A3G from African green monkey (AGM), and AGM SIV Vif cannot neutralize A3G from humans and this has been recognized as a cross-species infection barrier (Bogerd et al., 2004; Mangeat et al., 2004; Schrofelbauer et al., 2004; Xu et al., 2004; Etienne et al., 2013; Letko et al., 2013). Initially, to identify the residues HIV Vif uses to interact with human A3G the human A3G amino acids were replaced with those of AGM or rhesus macaque A3G. Mutation of human $A 3 G{ }^{128} \mathrm{D}$ to ${ }^{128} \mathrm{~K}$ as found in AGM and rhesus macaque A3G abrogated the interaction of HIV Vif with human A3G and its ability to induce degradation of human A3G (Bogerd et al., 2004; Mangeat et al., 2004; Schrofelbauer et al., 2004). However, when the ${ }^{128} \mathrm{D}$ was mutated to ${ }^{128} \mathrm{~A}$, HIV Vif could still interact with and degrade human A3G demonstrating that the charged interface was more important than the amino acid identity (Schrofelbauer et al., 2004). Since mutation of solely ${ }^{128} \mathrm{D}$ to ${ }^{128} \mathrm{~K}$ can abrogate the interaction between $\mathrm{A} 3 \mathrm{G}$ and Vif in co-immunoprecipitation studies it is clearly a determining residue. However, Vif-mediated degradation can be influenced by mutation of $A 3 G{ }^{129} \mathrm{P}$ and ${ }^{130} \mathrm{D}$ and Vif also interacts with A3G on surrounding motifs such as helix 6 (Huthoff and Malim, 2007; Lavens et al., 2010; Feng et al., 2013, Figure 3A). The loop 7 and helix 6 regions contain more positively charged and neutral amino acids than negatively charged amino acids which may explain why ${ }^{128} \mathrm{D}$ is such an important contact point for the positively charged Vif, despite a larger A3G interface predicted from biochemical studies. These studies with $\mathrm{A} 3 \mathrm{G}$ established the principle that a lack of Vif-induced degradation correlates with a lack of an interaction between the $\mathrm{A} 3$ and Vif.

\section{Vif-A3F/A3D interaction}

$\mathrm{A} 3 \mathrm{~F}$ and $\mathrm{A} 3 \mathrm{D}$ share the same structural motif in the CTD that interacts with Vif (Smith and Pathak, 2010; Kitamura et al., 2012, Figures 3D,E). A3F has been studied more extensively than A3D in this regard and will be discussed here. In contrast to A3G, there was no specific single amino acid determinant identified for A3F that clearly mediated both the primary interaction with Vif and was a determinant for Vif-mediated degradation. Rather, different groups identified different amino acids in A3F that altered its susceptibility to Vif. Smith and Pathak (2010) reported that A3F interacts with Vif through CTD amino acids ${ }^{289} \mathrm{EFLARH}^{294}$ and that ${ }^{289} \mathrm{E}$ was critical for A3F sensitivity to Vif. Albin et al. (2010b) identified another residue, ${ }^{324} \mathrm{E}$, as the key determinant of $\mathrm{A} 3 \mathrm{~F}$ to Vif-mediated degradation, but mutation of ${ }^{324} \mathrm{E}$ to other amino acids, even those of opposite charge, did not disrupt the interaction between $\mathrm{A} 3 \mathrm{~F}$ and Vif under stringent co-immunoprecipitation conditions. Although other groups have found that the interaction of A3F and Vif could be disrupted at least partially by mutating ${ }^{324} \mathrm{E}$, there was a wider region of $\mathrm{A} 3 \mathrm{~F}$ that appeared to be important for Vif-mediated degradation in comparison to what was identified for A3G (Albin et al., 2010b; Kitamura et al., 2012; Siu et al., 2013). A combination of mutagenesis, structural modeling and a 
crystal structure of $\mathrm{A} 3 \mathrm{C}$, that shares the same Vif binding interface with $\mathrm{A} 3 \mathrm{~F}$ and $\mathrm{A} 3 \mathrm{D}$, identified a novel type of $\mathrm{A} 3$ and Vif interaction (Smith and Pathak, 2010; Kitamura et al., 2012). Rather than Vif interacting with a loop as in the case of A3G (Figure 3A, loop 7), Vif interacted with a negatively charged surface of A3F/A3D/A3C that spanned helix 2, 3, and 4 and $\beta$-strand 4 (Kitamura et al., 2012; Aydin et al., 2014, Figures 3D,E). This negatively charged surface supports the hypothesis that it is primarily electrostatic interactions that mediated the $\mathrm{A} 3$ and Vif interaction and provides an explanation for why the $\mathrm{A} 3 \mathrm{~F}$ and $\mathrm{Vif}$ interaction may be more difficult to disrupt than the primarily neutral surface present in A3G. It is not known if this would mediate a tighter interaction of Vif with $\mathrm{A} 3 \mathrm{~F}$ than $\mathrm{A} 3 \mathrm{G}$ since there are no quantitative data available for both A3G and A3F using the same experimental conditions (Feng et al., 2013; Siu et al., 2013). Studies with A3F have shown that a lack of Vif-induced degradation does not necessarily correlate with a lack of a Vif-A3F interaction, suggesting that the binding orientation or other factors contribute to successful Vif-mediated degradation rather than only the presence of an interaction (Albin et al., 2010b).

\section{Vif-A3H interaction}

A3H sensitivity to Vif is haplotype dependent (OhAinle et al., 2008; Harari et al., 2009; Tan et al., 2009; Li et al., 2010; Hultquist et al., 2011; Binka et al., 2012). The A3H Hap I is not sensitive to HIV LAI Vif-mediated degradation whereas A3H Hap II is sensitive to HIV LAI Vif-mediated degradation (Harari et al., 2009; Li et al., 2010; Zhen et al., 2010; Ooms et al., 2013b). The A3H haplotype polymorphisms only occur at three locations (amino acids 105, 121, and 178, Table 1). A3H Hap I encodes GKE at these three positions and $\mathrm{A} 3 \mathrm{H}$ Hap II encodes RDD at these positions. It was shown that at position 105, the Arg is required for stable expression in cells and that the 178 position had little effect on Vif-mediated degradation (OhAinle et al., 2008; Harari et al., 2009; Li et al., 2010). Therefore, a single amino acid homologous to $\mathrm{A} 3 \mathrm{G}{ }^{128} \mathrm{D}$ at position 121 in A3H Hap II was determined to control sensitivity to Vif-mediated degradation (Li et al., 2010; Zhen et al., 2010, Figure 3F). An A3H Hap II mutant with a ${ }^{121} \mathrm{~K}$ is not sensitive to Vif-mediated degradation and does not interact with Vif (Zhen et al., 2010). From the A3H structural model (Figure 3F), it appears that the ${ }^{121} \mathrm{D}$ of $\mathrm{A} 3 \mathrm{H}$ is not located on loop 7 as in A3G, but is on helix 4 and on a different face of the molecule (compare Figures 3A,F). Yet, similar to $A 3 G$ the region surrounding ${ }^{121} \mathrm{D}$ is mainly neutral or positively charged residues, in contrast to the negatively charged interface that Vif uses to interact with A3F and A3D (Aydin et al., 2014).

\section{DEGRADATION INDEPENDENT INHIBITION OF A3G}

Although Vif primarily inhibits A3G by inducing its proteasomal degradation, there have been other ways in which Vif can inhibit $\mathrm{A} 3 \mathrm{G}$ encapsidation or function through a degradationindependent route. Vif may not be able to completely degrade the $\mathrm{A} 3 \mathrm{G}$ in the virus-producing cell and these degradationindependent mechanisms may be another line of defense against $A 3 G$ virion encapsidation. In particular, Vif can become the target of A3-mediated hypermutation (Simon et al., 2005; Jern et al., 2009), which may result in a Vif unable to interact with the E3 CRL5 ligase complex, but still able to inhibit A3G through a degradation-independent mechanism. It is not known if Vif can act in this manner for other A3 enzymes.

\section{Vif decreases translation of A3G mRNA}

Vif can decrease A3G mRNA translation in order to lower the steady-state levels of A3G through a Vif and A3G mRNA interaction, but the exact mechanism is not understood (Kao et al., 2003; Stopak et al., 2003; Mercenne et al., 2010). It is known that Vif can decrease the mRNA levels of $\mathrm{A} 3 \mathrm{G}$ by $15-40 \%$ and this requires that Vif interact with the $5^{\prime} \mathrm{UTR}$ of the A3G mRNA (Stopak et al., 2003; Mercenne et al., 2010). Since Vif has been shown in an immunofluorescence study to co-localize with A3 enzymes and P-bodies (Marin et al., 2008), it is possible that Vif shuttles A3G mRNA to P-bodies to delay or prevent mRNA translation.

\section{Vif inhibits virion encapsidation of A3G}

Studies by Goila-Gaur et al. (2008) have shown that A3G synthesized in vitro using a rabbit reticulocyte lysate translation system would become immunoprecipitation and packaging incompetent in the presence of Vif. Vif was not associated with these high molecular mass A3G forms, but was required for their formation (Goila-Gaur et al., 2008). Although A3G regularly forms high molecular mass complexes in cells, which are less likely to be packaged into virions, Vif can induce an even higher molecular weight form of A3G (Soros et al., 2007; Goila-Gaur et al., 2008). Moreover, studies with an A3G C97A mutant that is resistant to Vif-mediated degradation suggested that Vif-mediated degradation and inhibition of packaging are two distinct properties of A3G since the A3G C97A mutant was encapsidated less well in the presence of Vif (Opi et al., 2007). A molecular mechanism for this effect has not been described.

\section{Vif inhibits deamination of deoxycytidine by virion-encapsidated A3G}

A3 enzymes are mainly studied with HIV $\Delta$ vif in order to observe restriction in single-cycle infectivity assays, but in infected individuals A3 enzymes must contend with Vif. Despite multiple mechanisms that Vif uses to block A3G, it has been shown that A3G is encapsidated in the presence of Vif, albeit in lesser amounts (Nowarski et al., 2008). However, per molecule of A3G there is less deamination activity (Britan-Rosich et al., 2011, Figure 2E). This decrease in A3G deamination activity even occurs when A3G and Vif are coexpressed in E. coli and mutations are detected with a Rifampicin reversion assay or in vitro with purified A3G and Vif, demonstrating that other viral components are not required for the inhibition to take place (Santa-Marta et al., 2005; Britan-Rosich et al., 2011; Feng et al., 2013). Enzymatic studies have shown that Vif can cause a decrease in the specific activity of A3G and that this is due to a combination of Vif competitively binding to the ssDNA substrate and Vif binding directly to A3G (Britan-Rosich et al., 2011; Feng et al., 2013). These are separable functions of Vif since ssDNA-binding studies of the Vif-A3G complex in comparison to each of the components binding ssDNA alone support the hypothesis that Vif bound to A3G is unable to bind ssDNA with high affinity (Feng et al., 2013). Another consequence of Vif binding to $A 3 G$ is that it disrupts how A3G scans ssDNA in search of cytosines to deaminate (Feng et al., 2013). Vif interacts with the A3G NTD on loop 7, which is required for processive jumping 
movements (Feng and Chelico, 2011, Figure 3A). In a study that used two Vif variants to examine the effect of Vif/CBF $\beta$ on the deamination activity of A3G it was found that HIV HXB2 Vif inhibited A3G jumping movements, consistent with an interaction of Vif on loop 7 (Feng et al., 2013). In contrast, HIV NL4-3 Vif inhibited A3G sliding, which is mediated by helix 6, providing functional evidence that beyond the key loop 7 contact residues, ${ }^{128} \mathrm{DPD}^{130}$, Vif variants can interact with different regions of A3G (Feng et al., 2013). This appears to have no functional consequence for A3G-mediated degradation (Binka et al., 2012), but provides insights on how variable the Vif variants can be in their extended binding sites on A3 enzymes. This may affect strategies that aim to use small molecule inhibitors of the Vif-A3 interaction as an HIV therapy. Importantly, it has been shown that the specific activity of an A3 enzyme is not of primary importance for high levels of deoxycytidine deamination during reverse transcription (Feng et al., 2013; Ara etal., 2014). Rather, the method of efficiently searching for the cytosines, i.e., the enzyme's processive mechanism appears to be of more importance (Feng et al., 2013; Ara et al., 2014). All together the data suggest that the mechanism by which Vif inhibits A3G deamination activity in virions is by altering the searching mechanism used to find cytosines on ssDNA. Inhibition of A3G deaminase activity by Vif is likely to result in sublethal mutagenesis of HIV and could contribute to the generation of viral quasispecies and HIV evolution (Sadler et al., 2010; Feng et al., 2013).

\section{DEVELOPMENT OF SMALL-MOLECULE INHIBITORS FOR A3-BASED HIV THERAPEUTICS}

The A3-HIV host and pathogen relationship creates the possibility of developing novel therapeutics (Greene et al., 2008; Albin and Harris, 2010) and high-throughput screening approaches for small-molecule inhibitors have uncovered positive results. There are strategies to induce either A3G-mediated viral hypermutation by disrupting the Vif-A3G interaction (Nathans et al., 2008; Cen et al., 2010; Nowotny et al., 2010; Ejima et al., 2011; Ali et al., 2012; Mohammed et al., 2012; Matsui et al., 2014a) or viral hypomutation by blocking A3G catalytic activity (Li et al., 2012; Olson et al., 2013). For the "therapy by hypermutation" strategy, the rationale is to find small-molecule inhibitors that antagonize Vif function and increase the cellular level of A3G available for virus restriction. A few candidate molecules that recover A3G expression levels and enable HIV restriction in the presence of Vif have been discovered (Nathans et al., 2008; Cen et al., 2010; Nowotny et al., 2010; Ejima et al., 2011; Ali et al., 2012; Mohammed et al., 2012; Matsui et al., 2014a), although there are little biochemical data to understand the mechanism of action. For the "therapy by hypomutation" strategy, small-molecule inhibitors have been designed that target a key residue in $\mathrm{A} 3 \mathrm{G}$ ( $\mathrm{C} 321)$ that inhibits the catalytic activity of A3G (Li et al., 2012; Olson et al., 2013). It is thought that decreasing the viral quasispecies that may arise due to A3-mediated mutagenesis can assist in immune clearance of the virus and decrease resistance to antivirals (Harris, 2008; Hultquist and Harris, 2009).

Inhibitors targeted to Vif may only be successful if administered in a cocktail to cycle their use and prevent the development of drug resistance, a long-standing therapy regimen for HIV-1 drugs (Greene, 2007). One strategy to avoid selection of resistant
Vif variants is to utilize the anti-HIV potential of each A3 enzyme and design inhibitors that bind different regions of Vif, based on the unique interactions that Vif has with $\mathrm{A} 3 \mathrm{G}, \mathrm{A} 3 \mathrm{~F} / \mathrm{D}$, and $\mathrm{A} 3 \mathrm{H}$ (Figure 5A). However, more study is required to determine if all A3 enzymes function equally well as individual restriction factors otherwise, the strategy may need to involve inhibiting degradation of all A3 enzymes together to enable a strong restriction pressure on HIV. Development of inhibitors that target the A3 enzymes may be a problematic route when considering A3G since Vif interacts with $A 3 G$ near the amino acid residues needed for virion incorporation, oligomerization, and processivity (Huthoff and Malim, 2007; Huthoff et al., 2009; Feng et al., 2013, Figure 3A). As a result, the inhibitor molecule may decrease A3G anti-HIV activity. It is unknown whether the activity of $\mathrm{A} 3 \mathrm{D}, \mathrm{A} 3 \mathrm{H}$, or $\mathrm{A} 3 \mathrm{~F}$ would be affected by this type of strategy. Furthermore, it has been shown in the case of A3G, that HIV can overcome the restriction pressure of $\mathrm{A} 3 \mathrm{G}$ by acquiring mutations in genetic sequences other than Vif in order to indirectly avoid A3G encapsidation (Hache et al., 2008, 2009).

If Vif were unable to interact with CRL5 E3 ligase complex components, the accelerated degradation of $\mathrm{A} 3 \mathrm{G}$ would be blocked. This strategy has been raised as a potential option (Greene et al., 2008; Bergeron et al., 2010; Zuo et al., 2012) and current structural data on the EloB/C-, Cul5-, and CBF $\beta$ - Vif interfaces could facilitate development of inhibitors (Stanley et al., 2008; Guo et al., 2014). However, the consequence of targeting the host proteins with small molecules remains unknown. In addition, this approach has potential drawbacks since Vif may remain bound to the A3 enzymes. For A3G, this has been shown to lead to a decrease in mutagenic activity (Britan-Rosich et al., 2011; Feng et al., 2013). There are no published studies investigating whether Vif would affect the mutagenic activity of other A3s. If Vif were unable to interact with CBF $\beta$ it would become unstable in the host cells and degradation of A3 enzymes would be circumvented (Jager et al., 2012; Zhang et al., 2012). However, targeting a small molecule to $\mathrm{CBF} \beta$ may be problematic if this prevents $\mathrm{CBF} \beta$ from functioning as the transcription cofactor for RUNX proteins. Although some reports show that Vif and RUNX1 interact with CBF $\beta$ on distinct surfaces (Hultquist et al., 2012; Zhang et al., 2012; Du et al., 2013b), Kim et al. (2013) demonstrated that Vif recruitment of CBF $\beta$ alters the transcriptional profile of the cell by preventing RUNX1 and $\mathrm{CBF} \beta$ association.

\section{PERSPECTIVES}

A3 enzymes have the potential to be manipulated as a therapeutic mechanism to suppress HIV replication. Over the past decade, an immense amount of information has been learned regarding each individual A3 enzyme. Cellular, biochemical, and structural data have provided insights on how A3 enzymes interact with nucleic acids and Vif and these data can be strategically applied to develop novel therapies. Critical to predicting the success of an A3-based strategy requires long-term culture of the virus with the potential small molecules to identify tactics HIV could use to overcome the suppression. Another critical facet is understanding if it is necessary for A3 enzymes to work together to restrict HIV in vivo in order to invoke the most restrictive pressure on the virus and prevent sublethal mutagenesis. 


\section{REFERENCES}

Abdel-Mohsen, M., Raposo, R. A., Deng, X., Li, M., Liegler, T., Sinclair, E., et al. (2013). Expression profile of host restriction factors in HIV-1 elite controllers. Retrovirology 10, 106. doi: 10.1186/1742-4690-10-106

Adolph, M. B., Webb, J., and Chelico, L. (2013). Retroviral restriction factor APOBEC3G delays the initiation of DNA synthesis by HIV-1 reverse transcriptase. PLoS ONE 8:e64196. doi: 10.1371/journal.pone.0064196

Albin, J. S., Anderson, J. S., Johnson, J. R., Harjes, E., Matsuo, H., Krogan, N. J., etal. (2013). Dispersed sites of HIV Vif-dependent polyubiquitination in the DNA deaminase APOBEC3F. J. Mol. Biol. 425, 1172-1182. doi: 10.1016/j.jmb.2013.01.010

Albin, J. S., Brown, W. L., and Harris, R. S. (2014). Catalytic activity of APOBEC3F is required for efficient restriction of Vif-deficient human immunodeficiency virus. Virology 450-451, 49-54. doi: 10.1016/j.virol.2013.11.041

Albin, J. S., Hache, G., Hultquist, J. F., Brown, W. L., and Harris, R. S. (2010a). Long-term restriction by APOBEC3F selects human immunodeficiency virus type 1 variants with restored Vif function. J. Virol. 84, 10209-10219. doi: 10.1128/JVI.00632-10

Albin, J. S., Larue, R. S., Weaver, J. A., Brown, W. L., Shindo, K., Harjes, E., et al. (2010b). A single amino acid in human APOBEC3F alters susceptibility to HIV-1 Vif. J. Biol. Chem. 285, 40785-40792. doi: 10.1074/jbc.M110.173161

Albin, J. S., and Harris, R. S. (2010). Interactions of host APOBEC3 restriction factors with HIV-1 in vivo: implications for therapeutics. Expert Rev. Mol. Med. 12, e4. doi: 10.1017/S1462399409001343

Alce, T. M., and Popik, W. (2004). APOBEC3G is incorporated into virus-like particles by a direct interaction with HIV-1 Gag nucleocapsid protein. J. Biol. Chem. 279, 34083-34086. doi: 10.1074/jbc.C400235200

Ali, A., Wang, J., Nathans, R. S., Cao, H., Sharova, N., Stevenson, M., et al. (2012). Synthesis and structure-activity relationship studies of HIV-1 virion infectivity factor (Vif) inhibitors that block viral replication. ChemMedChem 7, 1217-1229. doi: 10.1002/cmdc.201200079

Ara, A., Love, R. P., and Chelico, L. (2014). Different mutagenic potential of HIV-1 restriction factors APOBEC3G and APOBEC3F is determined by distinct single-stranded DNA scanning mechanisms. PLoS Pathog. 10:e1004024. doi: 10.1371/journal.ppat.1004024

Armitage, A. E., Katzourakis, A., De Oliveira, T., Welch, J. J., Belshaw, R., Bishop, K. N., et al. (2008). Conserved footprints of APOBEC3G on Hypermutated human immunodeficiency virus type 1 and human endogenous retrovirus HERV-K(HML2) sequences. J. Virol. 82, 8743-8761. doi: 10.1128/JVI.00584-08

Aydin, H., Taylor, M. W., and Lee, J. E. (2014). Structure-guided analysis of the human APOBEC3-HIV restrictome. Structure 22, 668-684. doi: 10.1016/j.str.2014.02.011

Bach, D., Peddi, S., Mangeat, B., Lakkaraju, A., Strub, K., and Trono, D. (2008). Characterization of APOBEC3G binding to 7SL RNA. Retrovirology 5, 54. doi: 10.1186/1742-4690-5-54

Batisse, J., Guerrero, S., Bernacchi, S., Sleiman, D., Gabus, C., Darlix, J. L., et al. (2012). The role of Vif oligomerization and RNA chaperone activity in HIV-1 replication. Virus Res. 169, 361-376. doi: 10.1016/j.virusres.2012.06.018

Belanger, K., Savoie, M., Rosales Gerpe, M. C., Couture, J. F., and Langlois, M. A. (2013). Binding of RNA by APOBEC3G controls deaminationindependent restriction of retroviruses. Nucleic Acids Res. 41, 7438-7452. doi: 10.1093/nar/gkt527

Berg, O. G., Winter, R. B., and Von Hippel, P. H. (1981). Diffusion-driven mechanisms of protein translocation on nucleic acids. 1. Models and theory. Biochemistry 20, 6929-6948. doi: 10.1021/bi00527a028

Berger, G., Durand, S., Fargier, G., Nguyen, X. N., Cordeil, S., Bouaziz, S., et al. (2011). APOBEC3A is a specific inhibitor of the early phases of HIV-1 infection in myeloid cells. PLoS Pathog. 7:e1002221. doi: 10.1371/journal.ppat.1002221

Bergeron, J. R., Huthoff, H., Veselkov, D. A., Beavil, R. L., Simpson, P. J., Matthews, S. J., et al. (2010). The SOCS-box of HIV-1 Vif interacts with ElonginBC by induced-folding to recruit its Cul5-containing ubiquitin ligase complex. PLoS Pathog. 6:e1000925. doi: 10.1371/journal.ppat.1000925

Biasin, M., Piacentini, L., Lo Caputo, S., Kanari, Y., Magri, G., Trabattoni, D., et al. (2007). Apolipoprotein B mRNA-editing enzyme, catalytic polypeptide-like 3G: a possible role in the resistance to HIV of HIV-exposed seronegative individuals. J. Infect. Dis. 195, 960-964. doi: 10.1086/511988

Binka, M., Ooms, M., Steward, M., and Simon, V. (2012). The activity spectrum of Vif from multiple HIV-1 subtypes against APOBEC3G,
APOBEC3F, and APOBEC3H. J. Virol. 86, 49-59. doi: 10.1128/JVI. 06082-11

Bishop, K. N., Holmes, R. K., and Malim, M. H. (2006). Antiviral potency of APOBEC proteins does not correlate with cytidine deamination. J. Virol. 80, 8450-8458. doi: 10.1128/JVI.00839-06

Bishop, K. N., Holmes, R. K., Sheehy, A. M., Davidson, N. O., Cho, S. J., and Malim, M. H. (2004). Cytidine deamination of retroviral DNA by diverse APOBEC proteins. Curr. Biol. 14, 1392-1396. doi: 10.1016/j.cub.2004. 06.057

Bishop, K. N., Verma, M., Kim, E. Y., Wolinsky, S. M., and Malim, M. H. (2008). APOBEC3G inhibits elongation of HIV-1 reverse transcripts. PLoS Pathog. 4:e1000231. doi: 10.1371/journal.ppat.1000231

Blanc, D., Patience, C., Schulz, T. F., Weiss, R., and Spire, B. (1993). Transcomplementation of VIF- HIV-1 mutants in CEM cells suggests that VIF affects late steps of the viral life cycle. Virology 193, 186-192. doi: 10.1006/viro.1993.1114

Bogerd, H. P., and Cullen, B. R. (2008). Single-stranded RNA facilitates nucleocapsid: APOBEC3G complex formation. RNA 14, 1228-1236. doi: 10.1261/rna.964708

Bogerd, H. P., Doehle, B. P., Wiegand, H. L., and Cullen, B. R. (2004). A single amino acid difference in the host APOBEC3G protein controls the primate species specificity of HIV type 1 virion infectivity factor. Proc. Natl. Acad. Sci. U.S.A. 101, 3770-3774. doi: 10.1073/pnas.0307713101

Bogerd, H. P., Wiegand, H. L., Doehle, B. P., and Cullen, B. R. (2007). The intrinsic antiretroviral factor APOBEC3B contains two enzymatically active cytidine deaminase domains. Virology 364, 486-493. doi: 10.1016/j.virol.2007. 03.019

Bogerd, H. P., Wiegand, H. L., Doehle, B. P., Lueders, K. K., and Cullen, B. R. (2006). APOBEC3A and APOBEC3B are potent inhibitors of LTR-retrotransposon function in human cells. Nucleic Acids Res. 34, 89-95. doi: 10.1093/nar/gkj416

Bohn, M. F., Shandilya, S. M., Albin, J. S., Kouno, T., Anderson, B. D., Mcdougle, R. M., et al. (2013). Crystal structure of the DNA cytosine deaminase APOBEC3F: the catalytically active and HIV-1 Vif-binding domain. Structure 21, 1042-1050. doi: 10.1016/j.str.2013.04.010

Bonvin, M., and Greeve, J. (2007). Effects of point mutations in the cytidine deaminase domains of APOBEC3B on replication and hypermutation of hepatitis B virus in vitro. J. Gen. Virol. 88, 3270-3274. doi: 10.1099/vir.0.83149-0

Britan-Rosich, E., Nowarski, R., and Kotler, M. (2011). Multifaceted counterAPOBEC3G mechanisms employed by HIV-1 Vif. J. Mol. Biol. 410, 1065-1076. doi: 10.1016/j.jmb.2011.03.058

Browne, E. P., Allers, C., and Landau, N. R. (2009). Restriction of HIV-1 by APOBEC3G is cytidine deaminase-dependent. Virology 387, 313-321. doi: 10.1016/j.virol.2009.02.026

Bulliard, Y., Turelli, P., Rohrig, U. F., Zoete, V., Mangeat, B., Michielin, O., et al. (2009). Functional analysis and structural modeling of human APOBEC3G reveal the role of evolutionarily conserved elements in the inhibition of human immunodeficiency virus type 1 infection and Alu transposition. J. Virol. 83, 12611-12621. doi: 10.1128/JVI.01491-09

Burdick, R. C., Hu, W. S., and Pathak, V. K. (2013). Nuclear import of APOBEC3Flabeled HIV-1 preintegration complexes. Proc. Natl. Acad. Sci. U.S.A. 110, E4780E4789. doi: 10.1073/pnas.1315996110

Burnett, A., and Spearman, P. (2007). APOBEC3G multimers are recruited to the plasma membrane for packaging into human immunodeficiency virus type 1 virus-like particles in an RNA-dependent process requiring the NC basic linker. J. Virol. 81, 5000-5013. doi: 10.1128/JVI.02237-06

Byeon, I. J., Ahn, J., Mitra, M., Byeon, C. H., Hercik, K., Hritz, J., et al. (2013). NMR structure of human restriction factor APOBEC3A reveals substrate binding and enzyme specificity. Nat. Commun. 4, 1890. doi: 10.1038/ncomms 2883

Camaur, D., and Trono, D. (1996). Characterization of human immunodeficiency virus type 1 Vif particle incorporation. J. Virol. 70, 6106-6111.

Carpenter, M. A., Rajagurubandara, E., Wijesinghe, P., and Bhagwat, A. S. (2010). Determinants of sequence-specificity within human AID and APOBEC3G. DNA Repair (Amst) 9, 579-587. doi: 10.1016/j.dnarep.2010.02.010

Casartelli, N., Guivel-Benhassine, F., Bouziat, R., Brandler, S., Schwartz, O., and Moris, A. (2010). The antiviral factor APOBEC3G improves CTL recognition of cultured HIV-infected T cells. J. Exp. Med. 207, 39-49. doi: 10.1084/jem.20091933 Cen, S., Guo, F., Niu, M., Saadatmand, J., Deflassieux, J., and Kleiman, L. (2004). The interaction between HIV-1 Gag and APOBEC3G. J. Biol. Chem. 279, 3317733184. doi: 10.1074/jbc.M402062200 
Cen, S., Peng, Z. G., Li, X. Y., Li, Z. R., Ma, J., Wang, Y. M., et al. (2010). Small molecular compounds inhibit HIV-1 replication through specifically stabilizing APOBEC3G. J. Biol. Chem. 285, 16546-16552. doi: 10.1074/jbc.M109. 085308

Chaipan, C., Smith, J. L., Hu, W. S., and Pathak, V. K. (2013). APOBEC3G restricts HIV-1 to a greater extent than APOBEC3F and APOBEC3DE in human primary CD4+ T cells and macrophages. J. Virol. 87, 444-453. doi: 10.1128/JVI.00676-12

Chaurasiya, K. R., Mccauley, M. J., Wang, W., Qualley, D. F., Wu, T., Kitamura, S., et al. (2014). Oligomerization transforms human APOBEC3G from an efficient enzyme to a slowly dissociating nucleic acid-binding protein. Nat. Chem. 6, 28-33. doi: $10.1038 /$ nchem.1795

Chelico, L., Pham, P., Calabrese, P., and Goodman, M. F. (2006). APOBEC3G DNA deaminase acts processively $3^{\prime} \rightarrow 5^{\prime}$ on single-stranded DNA. Nat. Struct. Mol. Biol. 13, 392-399. doi: 10.1038/nsmb1086

Chelico, L., Pham, P., and Goodman, M. F. (2009). Mechanisms of APOBEC3Gcatalyzed processive deamination of deoxycytidine on single-stranded DNA. Nat. Struct. Mol. Biol. 16, 454-455. doi: 10.1038/nsmb0509-454

Chelico, L., Prochnow, C., Erie, D. A., Chen, X. S., and Goodman, M. F. (2010). Structural model for deoxycytidine deamination mechanisms of the HIV-1 inactivation enzyme APOBEC3G. J. Biol. Chem. 285, 16195-16205. doi: 10.1074/jbc.M110.107987

Chelico, L., Sacho, E. J., Erie, D. A., and Goodman, M. F. (2008). A model for oligomeric regulation of APOBEC3G cytosine deaminase-dependent restriction of HIV. J. Biol. Chem. 283, 13780-13791. doi: 10.1074/jbc.M801004200

Chen, G., He, Z., Wang, T., Xu, R., and Yu, X. F. (2009). A patch of positively charged amino acids surrounding the human immunodeficiency virus type 1 Vif SLVx4Yx9Y motif influences its interaction with APOBEC3G. J. Virol. 83, 8674-8682. doi: 10.1128/JVI.00653-09

Chiu, Y. L., Witkowska, H. E., Hall, S. C., Santiago, M., Soros, V. B., Esnault, C., et al. (2006). High-molecular-mass APOBEC3G complexes restrict Alu retrotransposition. Proc. Natl. Acad. Sci. U.S.A. 103, 15588-15593. doi: 10.1073/pnas.0604524103

Coffin, J., Hughes, S., and Varmus, H. (eds). (1997). Retroviruses. Cold Spring Harbor, NY: Cold Spring Harbor Laboratory Press.

Compton, A. A., and Emerman, M. (2013). Convergence and divergence in the evolution of the APOBEC3G-Vif interaction reveal ancient origins of simian immunodeficiency viruses. PLoS Pathog. 9:e1003135. doi: 10.1371/journal.ppat. 1003135

Conticello, S. G., Harris, R. S., and Neuberger, M. S. (2003). The Vif protein of HIV triggers degradation of the human antiretroviral DNA deaminase APOBEC3G. Curr. Biol. 13, 2009-2013. doi: 10.1016/j.cub.2003.10.034

Dang, Y., Abudu, A., Son, S., Harjes, E., Spearman, P., Matsuo, H., et al. (2011). Identification of a single amino acid required for APOBEC3 antiretroviral cytidine deaminase activity. J. Virol. 85, 5691-5695. doi: 10.1128/JVI.00243-11

Dang, Y., Siew, L. M., Wang, X., Han, Y., Lampen, R., and Zheng, Y. H. (2008). Human cytidine deaminase APOBEC3H restricts HIV-1 replication. J. Biol. Chem. 283 11606-11614. doi: 10.1074/jbc.M707586200

Dang, Y., Wang, X., Esselman, W. J., and Zheng, Y. H. (2006). Identification of APOBEC3DE as another antiretroviral factor from the human APOBEC family. J. Virol. 80, 10522-10533. doi: 10.1128/JVI.01123-06

Dang, Y., Wang, X., Zhou, T., York, I. A., and Zheng, Y. H. (2009). Identification of a novel WxSLVK motif in the $\mathrm{N}$ terminus of human immunodeficiency virus and simian immunodeficiency virus Vif that is critical for APOBEC3G and APOBEC3F neutralization. J. Virol. 83, 8544-8552. doi: 10.1128/JVI.00651-09

Doehle, B. P., Schafer, A., and Cullen, B. R. (2005). Human APOBEC3B is a potent inhibitor of HIV-1 infectivity and is resistant to HIV-1 Vif. Virology 339, 281-288. doi: 10.1016/j.virol.2005.06.005

Douaisi, M., Dussart, S., Courcoul, M., Bessou, G., Vigne, R., and Decroly, E. (2004). HIV-1 and MLV Gag proteins are sufficient to recruit APOBEC3G into virus-like particles. Biochem. Biophys. Res. Commun. 321, 566-573. doi: 10.1016/j.bbrc.2004.07.005

Du, J., Wu, X., Long, F., Wen, J., Hao, W., Chen, R., et al. (2013a). Improvement in efficacy of DNA vaccine encoding HIV-1 Vif by LIGHT gene adjuvant. Viral Immunol. 26, 68-74. doi: 10.1089/vim.2012.0073

Du, J., Zhao, K., Rui, Y., Li, P., Zhou, X., Zhang, W., et al. (2013b). Differential requirements for HIV-1 Vif-mediated APOBEC3G degradation and RUNX1mediated transcription by core binding factor beta. J. Virol. 87, 1906-1911. doi: 10.1128/JVI.02199-12
Duggal, N. K., Fu, W., Akey, J. M., and Emerman, M. (2013). Identification and antiviral activity of common polymorphisms in the APOBEC3 locus in human populations. Virology 443, 329-337. doi: 10.1016/j.virol.2013.05.016

Duggal, N. K., Malik, H. S., and Emerman, M. (2011). The breadth of antiviral activity of Apobec3DE in chimpanzees has been driven by positive selection. J. Virol. 85, 11361-11371. doi: 10.1128/JVI.05046-11

Ejima, T., Hirota, M., Mizukami, T., Otsuka, M., and Fujita, M. (2011). An anti-HIV1 compound that increases steady-state expression of apoplipoprotein B mRNAediting enzyme-catalytic polypeptide-like 3G. Int. J. Mol. Med. 28, 613-616. doi: 10.3892/ijmm.2011.737

Esnault, C., Heidmann, O., Delebecque, F., Dewannieux, M., Ribet, D., Hance, A. J., et al. (2005). APOBEC3G cytidine deaminase inhibits retrotransposition of endogenous retroviruses. Nature 433, 430-433. doi: 10.1038/nature03238

Etienne, L., Hahn, B. H., Sharp, P. M., Matsen, F. A., and Emerman, M. (2013). Gene loss and adaptation to hominids underlie the ancient origin of HIV-1. Cell Host Microbe 14, 85-92. doi: 10.1016/j.chom.2013.06.002

Feng, Y., and Chelico, L. (2011). Intensity of deoxycytidine deamination of HIV-1 proviral DNA by the retroviral restriction factor APOBEC3G is mediated by the noncatalytic domain. J. Biol. Chem. 286, 11415-11426. doi: 10.1074/jbc.M110.199604

Feng, Y., Love, R. P., and Chelico, L. (2013). HIV-1 viral infectivity factor (Vif) alters processive single-stranded DNA scanning of the retroviral restriction factor APOBEC3G. J. Biol. Chem. 288, 6083-6094. doi: 10.1074/jbc.M112.421875

Fisher, A. G., Ensoli, B., Ivanoff, L., Chamberlain, M., Petteway, S., Ratner, L., et al. (1987). The sor gene of HIV-1 is required for efficient virus transmission in vitro. Science 237, 888-893. doi: 10.1126/science.3497453

Fitzgibbon, J. E., Mazar, S., and Dubin, D. T. (1993). A new type of G->A hypermutation affecting human immunodeficiency virus. AIDS Res. Hum. Retroviruses 9, 833-838. doi: 10.1089/aid.1993.9.833

Fouchier, R. A., Simon, J. H., Jaffe, A. B., and Malim, M. H. (1996). Human immunodeficiency virus type 1 Vif does not influence expression or virion incorporation of gag-, pol-, and env-encoded proteins. J. Virol. 70, 8263-8269.

Fourati, S., Malet, I., Binka, M., Boukobza, S., Wirden, M., Sayon, S., et al. (2010). Partially active HIV-1 Vif alleles facilitate viral escape from specific antiretrovirals. AIDS 24, 2313-2321. doi: 10.1097/QAD.0b013e32833e515a

Fribourgh, J. L., Nguyen, H. C., Wolfe, L. S., Dewitt, D. C., Zhang, W., Yu, X. F, et al. (2014). Core binding factor beta plays a critical role by facilitating the assembly of the Vif-cullin 5 E3 ubiquitin ligase. J. Virol. 88, 3309-3319. doi: 10.1128/JVI.03824-13

Gabuzda, D. H., Lawrence, K., Langhoff, E., Terwilliger, E., Dorfman, T., Haseltine, W. A., et al. (1992). Role of vif in replication of human immunodeficiency virus type 1 in CD4+ T lymphocytes. J. Virol. 66, 6489-6495.

Gallois-Montbrun, S., Holmes, R. K., Swanson, C. M., Fernandez-Ocana, M., Byers, H. L., Ward, M. A., et al. (2008). Comparison of cellular ribonucleoprotein complexes associated with the APOBEC3F and APOBEC3G antiviral proteins. J. Virol. 82, 5636-5642. doi: 10.1128/JVI.00287-08

Gillick, K., Pollpeter, D., Phalora, P., Kim, E. Y., Wolinsky, S. M., and Malim, M. H. (2013). Suppression of HIV-1 infection by APOBEC3 proteins in primary human $\mathrm{CD} 4(+) \mathrm{T}$ cells is associated with inhibition of processive reverse transcription as well as excessive cytidine deamination. J. Virol. 87, 1508-1517. doi: 10.1128/JVI.02587-12

Goila-Gaur, R., Khan, M. A., Miyagi, E., Kao, S., Opi, S., Takeuchi, H., et al. (2008). HIV-1 Vif promotes the formation of high molecular mass APOBEC3G complexes. Virology 372, 136-146. doi: 10.1016/j.virol.2007.10.017

Goila-Gaur, R., Khan, M. A., Miyagi, E., Kao, S., and Strebel, K. (2007). Targeting APOBEC3A to the viral nucleoprotein complex confers antiviral activity. Retrovirology 4, 61. doi: 10.1186/1742-4690-4-61

Gourraud, P. A., Karaouni, A., Woo, J. M., Schmidt, T., Oksenberg, J. R., Hecht, F. M., et al. (2011). APOBEC3H haplotypes and HIV-1 pro-viral vif DNA sequence diversity in early untreated human immunodeficiency virus-1 infection. Hum. Immunol. 72, 207-212. doi: 10.1016/j.humimm.2010.12.008

Greene, W. C. (2007). A history of AIDS: looking back to see ahead. Eur. J. Immunol. 37(Suppl. 1), S94-S102. doi: 10.1002/eji.200737441

Greene, W. C., Debyser, Z., Ikeda, Y., Freed, E. O., Stephens, E., Yonemoto, W., et al. (2008). Novel targets for HIV therapy. Antiviral Res. 80, 251-265. doi: 10.1016/j.antiviral.2008.08.003

Guo, F., Cen, S., Niu, M., Saadatmand, J., and Kleiman, L. (2006). Inhibition of formula-primed reverse transcription by human APOBEC3G during human 
immunodeficiency virus type 1 replication. J. Virol. 80, 11710-11722. doi: 10.1128/JVI.01038-06

Guo, F., Cen, S., Niu, M., Yang, Y., Gorelick, R. J., and Kleiman, L. (2007). The interaction of APOBEC3G with human immunodeficiency virus type 1 nucleocapsid inhibits tRNA3Lys annealing to viral RNA. J. Virol. 81, 11322-11331. doi: 10.1128/JVI.00162-07

Guo, Y., Dong, L., Qiu, X., Wang, Y., Zhang, B., Liu, H., et al. (2014). Structural basis for hijacking CBF-beta and CUL5 E3 ligase complex by HIV-1 Vif. Nature 505, 229-233. doi: 10.1038/nature12884

Hache, G., Abbink, T. E., Berkhout, B., and Harris, R. S. (2009). Optimal translation initiation enables Vif-deficient human immunodeficiency virus type 1 to escape restriction by APOBEC3G. J. Virol. 83, 5956-5960. doi: 10.1128/JVI.00045-09

Hache, G., Liddament, M. T., and Harris, R. S. (2005). The retroviral hypermutation specificity of APOBEC3F and APOBEC3G is governed by the C-terminal DNA cytosine deaminase domain. J. Biol. Chem. 280, 10920-10924. doi: 10.1074/jbc.M500382200

Hache, G., Shindo, K., Albin, J. S., and Harris, R. S. (2008). Evolution of HIV1 isolates that use a novel Vif-independent mechanism to resist restriction by human APOBEC3G. Curr. Biol. 18, 819-824. doi: 10.1016/j.cub.2008.04.073

Halford, S. E., and Marko, J. F. (2004). How do site-specific DNA-binding proteins find their targets? Nucleic Acids Res. 32, 3040-3052. doi: 10.1093/nar/gkh624

Hancks, D. C., and Kazazian, H. H. Jr. (2012). Active human retrotransposons: variation and disease. Curr. Opin. Genet. Dev. 22, 191-203. doi: 10.1016/j.gde.2012.02.006

Harari, A., Ooms, M., Mulder, L. C., and Simon, V. (2009). Polymorphisms and splice variants influence the antiretroviral activity of human APOBEC3H. J. Virol. 83, 295-303. doi: 10.1128/JVI.01665-08

Harris, R. S. (2008). Enhancing immunity to HIV through APOBEC. Nat Biotechnol. 26, 1089-1090. doi: 10.1038/nbt1008-1089

Harris, R. S., Bishop, K. N., Sheehy, A. M., Craig, H. M., Petersen-Mahrt, S. K., Watt, I. N., et al. (2003). DNA deamination mediates innate immunity to retroviral infection. Cell 113, 803-809. doi: 10.1016/S0092-8674(03)00423-9

Harris, R. S., Hultquist, J. F., and Evans, D. T. (2012). The restriction factors of human immunodeficiency virus. J. Biol. Chem. 287, 40875-40883. doi: 10.1074/jbc.R112.416925

He, Z., Zhang, W., Chen, G., Xu, R., and Yu, X. F. (2008). Characterization of conserved motifs in HIV-1 Vif required for APOBEC3G and APOBEC3F interaction. J. Mol. Biol. 381, 1000-1011. doi: 10.1016/j.jmb.2008.06.061

Henriet, S., Sinck, L., Bec, G., Gorelick, R. J., Marquet, R., and Paillart, J. C. (2007). Vif is a RNA chaperone that could temporally regulate RNA dimerization and the early steps of HIV-1 reverse transcription. Nucleic Acids Res. 35, 5141-5153. doi: 10.1093/nar/gkm542

Hoglund, S., Ohagen, A., Lawrence, K., and Gabuzda, D. (1994). Role of vif during packing of the core of HIV-1. Virology 201, 349-355. doi: 10.1006/viro.1994.1300

Holmes, R. K., Koning, F. A., Bishop, K. N., and Malim, M. H. (2007). APOBEC3F can inhibit the accumulation of HIV-1 reverse transcription products in the absence of hypermutation. Comparisons with APOBEC3G. J. Biol. Chem. 282, 2587-2595. doi: 10.1074/jbc.M607298200

Hu, C., Saenz, D. T., Fadel, H. J., Walker, W., Peretz, M., and Poeschla, E. M. (2010). The HIV-1 central polypurine tract functions as a second line of defense against APOBEC3G/F. J. Virol. 84, 11981-11993. doi: 10.1128/JVI.00723-10

Hultquist, J. F., Binka, M., Larue, R. S., Simon, V., and Harris, R. S. (2012). Vif proteins of human and simian immunodeficiency viruses require cellular CBFbeta to degrade APOBEC3 restriction factors. J. Virol. 86, 2874-2877. doi: 10.1128/JVI.06950-11

Hultquist, J. F., and Harris, R. S. (2009). Leveraging APOBEC3 proteins to alter the HIV mutation rate and combat AIDS. Future Virol. 4, 605. doi: 10.2217/fvl.09.59

Hultquist, J. F., Lengyel, J. A., Refsland, E. W., Larue, R. S., Lackey, L., Brown, W. L., et al. (2011). Human and rhesus APOBEC3D, APOBEC3F, APOBEC3G, and APOBEC3H demonstrate a conserved capacity to restrict Vif-deficient HIV-1. J. Virol. 85, 11220-11234. doi: 10.1128/JVI.05238-11

Huthoff, H., Autore, F., Gallois-Montbrun, S., Fraternali, F., and Malim, M. H. (2009). RNA-dependent oligomerization of APOBEC3G is required for restriction of HIV-1. PLoS Pathog. 5:e1000330. doi: 10.1371/journal.ppat.1000330

Huthoff, H., and Malim, M. H. (2007). Identification of amino acid residues in APOBEC $3 G$ required for regulation by human immunodeficiency virus type 1 Vif and Virion encapsidation. J. Virol. 81, 3807-3815. doi: 10.1128/JVI. 02795-06
Iwai, K., Yamanaka, K., Kamura, T., Minato, N., Conaway, R. C., Conaway, J. W., et al. (1999). Identification of the von Hippel-lindau tumor-suppressor protein as part of an active E3 ubiquitin ligase complex. Proc. Natl. Acad. Sci. U.S.A. 96, 12436-12441. doi: 10.1073/pnas.96.22.12436

Iwatani, Y., Chan, D. S., Wang, F., Maynard, K. S., Sugiura, W., Gronenborn, A. M., et al. (2007). Deaminase-independent inhibition of HIV-1 reverse transcription by APOBEC3G. Nucleic Acids Res. 35, 7096-7108. doi: 10.1093/nar/gkm750

Iwatani, Y., Takeuchi, H., Strebel, K., and Levin, J. G. (2006). Biochemical activities of highly purified, catalytically active human APOBEC3G: correlation with antiviral effect. J. Virol. 80, 5992-6002. doi: 10.1128/JVI.02680-05

Jager, S., Cimermancic, P., Gulbahce, N., Johnson, J. R., Mcgovern, K. E., Clarke, S. C., et al. (2012). Global landscape of HIV-human protein complexes. Nature 481, 365-370. doi: 10.1038/nature10719

Jarmuz, A., Chester, A., Bayliss, J., Gisbourne, J., Dunham, I., Scott, J., et al. (2002). An anthropoid-specific locus of orphan C to U RNA-editing enzymes on chromosome 22. Genomics 79, 285-296. doi: 10.1006/geno.2002.6718

Jern, P., and Coffin, J. M. (2008). Host-retrovirus arms race: trimming the budget. Cell Host Microbe 4, 196-197. doi: 10.1016/j.chom.2008.08.008

Jern, P., Russell, R. A., Pathak, V. K., and Coffin, J. M. (2009). Likely role of APOBEC3G-mediated G-to-A mutations in HIV-1 evolution and drug resistance. PLoS Pathog. 5:e1000367. doi: 10.1371/journal.ppat.1000367

Jin, X., Brooks, A., Chen, H., Bennett, R., Reichman, R., and Smith, H. (2005). APOBEC3G/CEM15 (hA3G) mRNA levels associate inversely with human immunodeficiency virus viremia. J. Virol. 79, 11513-11516. doi: 10.1128/JVI.79.17.11513-11516.2005

Jonsson, S. R., Hache, G., Stenglein, M. D., Fahrenkrug, S. C., Andresdottir, V., and Harris, R. S. (2006). Evolutionarily conserved and non-conserved retrovirus restriction activities of artiodactyl APOBEC3F proteins. Nucleic Acids Res. 34, 5683-5694. doi: 10.1093/nar/gkl721

Kaiser, S. M., and Emerman, M. (2006). Uracil DNA glycosylase is dispensable for human immunodeficiency virus type 1 replication and does not contribute to the antiviral effects of the cytidine deaminase Apobec3G. J. Virol. 80, 875-882. doi: 10.1128/JVI.80.2.875-882.2006

Kamura, T., Maenaka, K., Kotoshiba, S., Matsumoto, M., Kohda, D., Conaway, R. C., et al. (2004). VHL-box and SOCS-box domains determine binding specificity for Cul2-Rbx1 and Cul5-Rbx2 modules of ubiquitin ligases. Genes Dev. 18, 30553065. doi: 10.1101/gad.1252404

Kamura, T., Sato, S., Haque, D., Liu, L., Kaelin, W. G. Jr., et al. (1998). The Elongin BC complex interacts with the conserved SOCS-box motif present in members of the SOCS, ras, WD-40 repeat, and ankyrin repeat families. Genes Dev. 12, 3872-3881. doi: 10.1101/gad.12.24.3872

Kao, S., Khan, M. A., Miyagi, E., Plishka, R., Buckler-White, A., and Strebel, K. (2003). The human immunodeficiency virus type 1 Vif protein reduces intracellular expression and inhibits packaging of APOBEC3G (CEM15), a cellular inhibitor of virus infectivity. J. Virol. 77, 11398-11407. doi: 10.1128/JVI.77.21.1139811407.2003

Khan, M. A., Goila-Gaur, R., Opi, S., Miyagi, E., Takeuchi, H., Kao, S., et al. (2007). Analysis of the contribution of cellular and viral RNA to the packaging of APOBEC3G into HIV-1 virions. Retrovirology 4, 48. doi: 10.1186/1742-4690-4-48 Khatua, A. K., Taylor, H. E., Hildreth, J. E., and Popik, W. (2010). Inhibition of LINE-1 and Alu retrotransposition by exosomes encapsidating APOBEC3G and APOBEC3F. Virology 400, 68-75. doi: 10.1016/j.virol.2010.01.021

Kim, D. Y., Kwon, E., Hartley, P. D., Crosby, D. C., Mann, S., Krogan, N. J., et al. (2013). CBFbeta stabilizes HIV Vif to counteract APOBEC3 at the expense of RUNX1 target gene expression. Mol. Cell. 49, 632-644. doi: 10.1016/j.molcel.2012.12.012

Kim, E. Y., Bhattacharya, T., Kunstman, K., Swantek, P., Koning, F. A., Malim, M. H., et al. (2010). Human APOBEC3G-mediated editing can promote HIV-1 sequence diversification and accelerate adaptation to selective pressure. J. Virol. 84, 10402-10405. doi: 10.1128/JVI.01223-10

Kitamura, S., Ode, H., and Iwatani, Y. (2011). Structural features of Antiviral APOBEC3 proteins are linked to their functional activities. Front. Microbiol. 2:258. doi: $10.3389 /$ fmicb.2011.00258

Kitamura, S., Ode, H., Nakashima, M., Imahashi, M., Naganawa, Y., Kurosawa, T., et al. (2012). The APOBEC3C crystal structure and the interface for HIV-1 Vif binding. Nat. Struct. Mol. Biol. 19, 1005-1010. doi: 10.1038/nsmb.2378

Kobayashi, M., Takaori-Kondo, A., Miyauchi, Y., Iwai, K., and Uchiyama, T. (2005). Ubiquitination of APOBEC3G by an HIV-1 Vif-Cullin5-Elongin B-Elongin C 
complex is essential for Vif function. J. Biol. Chem. 280, 18573-18578. doi: 10.1074/jbc.C500082200

Kobayashi, T., Koizumi, Y., Takeuchi, J. S., Misawa, N., Kimura, Y., Morita, S., et al. (2014). Quantification of deaminase activity-dependent and -independent restriction of HIV-1 replication mediated by APOBEC3F and APOBEC3G through experimental-mathematical investigation. J. Virol. 88, 5881-5887. doi: 10.1128/JVI.00062-14

Kock, J., and Blum, H. E. (2008). Hypermutation of hepatitis B virus genomes by APOBEC3G, APOBEC3C and APOBEC3H. J. Gen. Virol. 89, 1184-1191. doi: 10.1099/vir.0.83507-0

Kohli, R. M., Abrams, S. R., Gajula, K. S., Maul, R. W., Gearhart, P. J., and Stivers, J. T. (2009). A portable hot spot recognition loop transfers sequence preferences from APOBEC family members to activation-induced cytidine deaminase. J. Biol. Chem. 284, 22898-22904. doi: 10.1074/jbc.M109.025536

Koning, F. A., Goujon, C., Bauby, H., and Malim, M. H. (2011). Target cell-mediated editing of HIV-1 cDNA by APOBEC3 proteins in human macrophages. J. Virol. 85, 13448-13452. doi: 10.1128/JVI.00775-11

Koning, F. A., Newman, E. N., Kim, E. Y., Kunstman, K. J., Wolinsky, S. M., and Malim, M. H. (2009). Defining APOBEC3 expression patterns in human tissues and hematopoietic cell subsets. J. Virol. 83, 9474-9485. doi: 10.1128/JVI.01089-09

Koyama, T., Arias, J. F., Iwabu, Y., Yokoyama, M., Fujita, H., Sato, H., et al. (2013). APOBEC3G oligomerization is associated with the inhibition of both Alu and LINE-1 retrotransposition. PLoS ONE 8:e84228. doi: 10.1371/journal.pone. 0084228

Kozak, S. L., Marin, M., Rose, K. M., Bystrom, C., and Kabat, D. (2006). The antiHIV-1 editing enzyme APOBEC3G binds HIV-1 RNA and messenger RNAs that shuttle between polysomes and stress granules. J. Biol. Chem. 281, 29105-29119. doi: 10.1074/jbc.M601901200

Land, A. M., Ball, T. B., Luo, M., Pilon, R., Sandstrom, P., Embree, J. E., et al. (2008). Human immunodeficiency virus (HIV) type 1 proviral hypermutation correlates with CD4 count in HIV-infected women from Kenya. J. Virol. 82, 8172-8182. doi: 10.1128/JVI.01115-08

Langlois, M. A., Beale, R. C., Conticello, S. G., and Neuberger, M. S. (2005). Mutational comparison of the single-domained APOBEC3C and doubledomained APOBEC3F/G anti-retroviral cytidine deaminases provides insight into their DNA target site specificities. Nucleic Acids Res. 33, 1913-1923. doi: 10.1093/nar/gki343

LaRue, R. S., Andresdottir, V., Blanchard, Y., Conticello, S. G., Derse, D., Emerman, M., et al. (2009). Guidelines for naming nonprimate APOBEC3 genes and proteins. J. Virol. 83, 494-497. doi: 10.1128/JVI.01976-08

LaRue, R. S., Jonsson, S. R., Silverstein, K. A., Lajoie, M., Bertrand, D., El-Mabrouk, N., et al. (2008). The artiodactyl APOBEC3 innate immune repertoire shows evidence for a multi-functional domain organization that existed in the ancestor of placental mammals. BMC Mol. Biol. 9:104. doi: 10.1186/1471-2199-9-104

Lavens, D., Peelman, F., Van Der Heyden, J., Uyttendaele, I., Catteeuw, D., Verhee, A., et al. (2010). Definition of the interacting interfaces of Apobec3G and HIV-1 Vif using MAPPIT mutagenesis analysis. Nucleic Acids Res. 38, 1902-1912. doi: $10.1093 / \mathrm{nar} / \mathrm{gkp} 1154$

Letko, M., Silvestri, G., Hahn, B. H., Bibollet-Ruche, F., Gokcumen, O., Simon, V., et al. (2013). Vif proteins from diverse primate lentiviral lineages use the same binding site in APOBEC3G. J. Virol. 87, 11861-11871. doi: 10.1128/JVI.01944-13

Li, J., Chen, Y., Li, M., Carpenter, M. A., Mcdougle, R. M., Luengas, E. M., etal. (2014). APOBEC3 multimerization correlates with HIV-1 packaging and restriction activity in living cells. J. Mol. Biol. 426, 1296-1307. doi: 10.1016/j.jmb.2013.12.014

Li, M., Shandilya, S. M., Carpenter, M. A., Rathore, A., Brown, W. L., Perkins, A. L., et al. (2012). First-in-class small molecule inhibitors of the single-strand DNA cytosine deaminase APOBEC3G. ACS Chem. Biol. 7, 506-517. doi: $10.1021 / \mathrm{cb} 200440 \mathrm{y}$

Li, M. M., and Emerman, M. (2011). Polymorphism in human APOBEC3H affects a phenotype dominant for subcellular localization and antiviral activity. J. Virol. 85, 8197-8207. doi: 10.1128/JVI.00624-11

Li, M. M., Wu, L. I., and Emerman, M. (2010). The range of human APOBEC3H sensitivity to lentiviral Vif proteins. J. Virol. 84, 88-95. doi: 10.1128/JVI.01344-09

Li, Y., Kappes, J. C., Conway, J. A., Price, R. W., Shaw, G. M., and Hahn, B. H. (1991). Molecular characterization of human immunodeficiency virus type 1 cloned directly from uncultured human brain tissue: identification of replicationcompetent and -defective viral genomes. J. Virol. 65, 3973-3985.
Liddament, M. T., Brown, W. L., Schumacher, A. J., and Harris, R. S. (2004). APOBEC3F properties and hypermutation preferences indicate activity against HIV-1 in vivo. Curr. Biol. 14, 1385-1391. doi: 10.1016/j.cub.2004.06.050

Liu, S., Harada, B. T., Miller, J. T., Le Grice, S. F., and Zhuang, X. (2010). Initiation complex dynamics direct the transitions between distinct phases of early HIV reverse transcription. Nat. Struct. Mol. Biol. 17, 1453-1460. doi: 10.1038/nsmb.1937

Logue, E. C., Bloch, N., Dhuey, E., Zhang, R., Cao, P., Herate, C., et al. (2014). A DNA sequence recognition loop on APOBEC3A controls substrate Specificity. PLoS ONE 9:e97062. doi: 10.1371/journal.pone.0097062

Love, R. P., Xu, H., and Chelico, L. (2012). Biochemical analysis of hypermutation by the deoxycytidine deaminase APOBEC3A. J. Biol. Chem. 287, 30812-30822. doi: 10.1074/jbc.M112.393181

Lucifora, J., Xia, Y., Reisinger, F., Zhang, K., Stadler, D., Cheng, X., et al. (2014). Specific and nonhepatotoxic degradation of nuclear hepatitis B virus cccDNA. Science 343, 1221-1228. doi: 10.1126/science.1243462

Luo, K., Wang, T., Liu, B., Tian, C., Xiao, Z., Kappes, J., et al. (2007). Cytidine deaminases APOBEC3G and APOBEC3F interact with human immunodeficiency virus type 1 integrase and inhibit proviral DNA formation. J. Virol. 81, 7238-7248. doi: 10.1128/JVI.02584-06

Luo, K., Xiao, Z., Ehrlich, E., Yu, Y., Liu, B., Zheng, S., et al. (2005). Primate lentiviral virion infectivity factors are substrate receptors that assemble with cullin 5-E3 ligase through a HCCH motif to suppress APOBEC3G. Proc. Natl. Acad. Sci. U.S.A. 102, 11444-11449. doi: 10.1073/pnas.05024 40102

MacDuff, D. A., Demorest, Z. L., and Harris, R. S. (2009). AID can restrict L1 retrotransposition suggesting a dual role in innate and adaptive immunity. Nucleic Acids Res. 37, 1854-1867. doi: 10.1093/nar/gkp030

Madani, N., and Kabat, D. (1998). An endogenous inhibitor of human immunodeficiency virus in human lymphocytes is overcome by the viral Vif protein. J. Virol. 72, 10251-10255.

Mangeat, B., Turelli, P., Caron, G., Friedli, M., Perrin, L., and Trono, D. (2003). Broad antiretroviral defence by human APOBEC3G through lethal editing of nascent reverse transcripts. Nature 424, 99-103. doi: 10.1038/nature01709

Mangeat, B., Turelli, P., Liao, S., and Trono, D. (2004). A single amino acid determinant governs the species-specific sensitivity of APOBEC3G to Vif action. J. Biol. Chem. 279, 14481-14483. doi: 10.1074/jbc.C400060200

Mariani, R., Chen, D., Schrofelbauer, B., Navarro, F., Konig, R., Bollman, B., et al. (2003). Species-specific exclusion of APOBEC3G from HIV-1 virions by Vif. Cell 114, 21-31. doi: 10.1016/S0092-8674(03)00515-4

Marin, M., Golem, S., Rose, K. M., Kozak, S. L., and Kabat, D. (2008). Human immunodeficiency virus type 1 Vif functionally interacts with diverse APOBEC3 cytidine deaminases and moves with them between cytoplasmic sites of mRNA metabolism. J. Virol. 82, 987-998. doi: 10.1128/JVI.01078-07

Marin, M., Rose, K. M., Kozak, S. L., and Kabat, D. (2003). HIV-1 Vif protein binds the editing enzyme APOBEC3G and induces its degradation. Nat. Med. 9, 1398-1403. doi: 10.1038/nm946

Matsui, M., Shindo, K., Izumi, T., Io, K., Shinohara, M., Komano, J., et al. (2014a). Small molecules that inhibit Vif-induced degradation of APOBEC3G. Virol. J. 11, 122. doi: 10.1186/1743-422X-11-122

Matsui, Y., Shindo, K., Nagata, K., Io, K., Tada, K., Iwai, F., et al. (2014b). Defining HIV-1 Vif residues that interact with CBFbeta by site-directed mutagenesis. Virology 449, 82-87. doi: 10.1016/j.virol.2013.11.004

Mbisa, J. L., Barr, R., Thomas, J. A., Vandegraaff, N., Dorweiler, I. J., Svarovskaia, E. S., et al. (2007). Human immunodeficiency virus type 1 cDNAs produced in the presence of APOBEC3G exhibit defects in plus-strand DNA transfer and integration. J. Virol. 81, 7099-7110. doi: 10.1128/JVI.00272-07

Mbisa, J. L., Bu, W., and Pathak, V. K. (2010). APOBEC3F and APOBEC3G inhibit HIV-1 DNA integration by different mechanisms. J. Virol. 84, 5250-5259. doi: 10.1128/JVI.02358-09

Mehle, A., Goncalves, J., Santa-Marta, M., Mcpike, M., and Gabuzda, D. (2004). Phosphorylation of a novel SOCS-box regulates assembly of the HIV-1 Vif-Cul5 complex that promotes APOBEC3G degradation. Genes Dev. 18, 2861-2866. doi: $10.1101 / \mathrm{gad} .1249904$

Mehle, A., Wilson, H., Zhang, C., Brazier, A. J., Mcpike, M., Pery, E., et al. (2007). Identification of an APOBEC3G binding site in human immunodeficiency virus type 1 Vif and inhibitors of Vif-APOBEC3G binding. J. Virol. 81, 13235-13241. doi: 10.1128/JVI.00204-07 
Mercenne, G., Bernacchi, S., Richer, D., Bec, G., Henriet, S., Paillart, J. C., et al. (2010). HIV-1 Vif binds to APOBEC3G mRNA and inhibits its translation. Nucleic Acids Res. 38, 633-646. doi: 10.1093/nar/gkp1009

Miyagi, E., Brown, C. R., Opi, S., Khan, M., Goila-Gaur, R., Kao, S., et al. (2010). Stably expressed APOBEC3F has negligible antiviral activity. J. Virol. 84, 1106711075. doi: 10.1128/JVI.01249-10

Miyagi, E., Opi, S., Takeuchi, H., Khan, M., Goila-Gaur, R., Kao, S., et al. (2007). Enzymatically active APOBEC3G is required for efficient inhibition of human immunodeficiency virus type 1. J. Virol. 81, 13346-13353. doi: 10.1128/JVI.01361-07

Mohammed, I., Parai, M. K., Jiang, X., Sharova, N., Singh, G., Stevenson, M., et al. (2012). SAR and lead optimization of an HIV-1 Vif-APOBEC3G axis inhibitor ACS Med. Chem. Lett. 3, 465-469. doi: 10.1021/ml300037k

Monajemi, M., Woodworth, C. F., Zipperlen, K., Gallant, M., Grant, M. D., and Larijani, M. (2014). Positioning of APOBEC3G/F mutational hotspots in the human immunodeficiency virus genome favors reduced recognition by CD8 $+\mathrm{T}$ cells. PLoS ONE 9:e93428. doi: 10.1371/journal.pone.0093428

Mulder, L. C., Harari, A., and Simon, V. (2008). Cytidine deamination induced HIV-1 drug resistance. Proc. Natl. Acad. Sci. U.S.A. 105, 5501-5506. doi: 10.1073/pnas.0710190105

Mulder, L. C., Ooms, M., Majdak, S., Smedresman, J., Linscheid, C., Harari, A., et al. (2010). Moderate influence of human APOBEC3F on HIV-1 replication in primary lymphocytes. J. Virol. 84, 9613-9617. doi: 10.1128/JVI.02630-09

Munk, C., Willemsen, A., and Bravo, I. G. (2012). An ancient history of gene duplications, fusions and losses in the evolution of APOBEC3 mutators in mammals. BMC Evol. Biol. 12:71. doi: 10.1186/1471-2148-12-71

Nathans, R., Cao, H., Sharova, N., Ali, A., Sharkey, M., Stranska, R., et al. (2008). Small-molecule inhibition of HIV-1 Vif. Nat. Biotechnol. 26, 1187-1192. doi: 10.1038/nbt.1496

Navarro, F., Bollman, B., Chen, H., Konig, R., Yu, Q., Chiles, K., et al. (2005). Complementary function of the two catalytic domains of APOBEC3G. Virology 333, 374-386. doi: 10.1016/j.virol.2005.01.011

Newman, E. N., Holmes, R. K., Craig, H. M., Klein, K. C., Lingappa, J. R., Malim, M. H., etal. (2005). Antiviral function of APOBEC3G can be dissociated from cytidine deaminase activity. Curr. Biol. 15, 166-170. doi: 10.1016/j.cub.2004.12.068

Nowarski, R., Britan-Rosich, E., Shiloach, T., and Kotler, M. (2008). Hypermutation by intersegmental transfer of APOBEC3G cytidine deaminase. Nat. Struct. Mol. Biol. 15, 1059-1066. doi: 10.1038/nsmb.1495

Nowotny, B., Schneider, T., Pradel, G., Schirmeister, T., Rethwilm, A., and Kirschner, M. (2010). Inducible APOBEC3G-Vif double stable cell line as a high-throughput screening platform to identify antiviral compounds. Antimicrob. Agents Chemother. 54, 78-87. doi: 10.1128/AAC.00775-09

OhAinle, M., Kerns, J. A., Li, M. M., Malik, H. S., and Emerman, M. (2008). Antiretroelement activity of APOBEC3H was lost twice in recent human evolution. Cell Host Microbe 4, 249-259. doi: 10.1016/j.chom.2008.07.005

OhAinle, M., Kerns, J. A., Malik, H. S., and Emerman, M. (2006). Adaptive evolution and antiviral activity of the conserved mammalian cytidine deaminase APOBEC3H. J. Virol. 80, 3853-3862. doi: 10.1128/JVI.80.8.3853-3862.2006

Olson, M. E., Li, M., Harris, R. S., and Harki, D. A. (2013). Smallmolecule APOBEC3G DNA cytosine deaminase inhibitors based on a 4-amino-1,2,4-triazole-3-thiol scaffold. ChemMedChem 8, 112-117. doi: $10.1002 / \mathrm{cmdc} .201200411$

Ooms, M., Brayton, B., Letko, M., Maio, S. M., Pilcher, C. D., Hecht, F. M., et al. (2013a). HIV-1 Vif adaptation to human APOBEC3H haplotypes. Cell Host Microbe 14, 411-421. doi: 10.1016/j.chom.2013.09.006

Ooms, M., Letko, M., Binka, M., and Simon, V. (2013b). The resistance of human APOBEC3H to HIV-1 NL4-3 molecular clone is determined by a single amino acid in Vif. PLoS ONE 8:e57744. doi: 10.1371/journal.pone.0057744

Ooms, M., Majdak, S., Seibert, C. W., Harari, A., and Simon, V. (2010). The localization of APOBEC3H variants in HIV-1 virions determines their antiviral activity. J. Virol. 84, 7961-7969. doi: 10.1128/JVI.00754-10

Opi, S., Kao, S., Goila-Gaur, R., Khan, M. A., Miyagi, E., Takeuchi, H., et al. (2007). Human immunodeficiency virus type 1 Vif inhibits packaging and antiviral activity of a degradation-resistant APOBEC3G variant. J. Virol. 81, 8236-8246. doi: 10.1128/JVI.02694-06

Pace, C., Keller, J., Nolan, D., James, I., Gaudieri, S., Moore, C., et al. (2006). Population level analysis of human immunodeficiency virus type 1 hypermutation and its relationship with APOBEC3G and vif genetic variation. J. Virol. 80, 9259-9269. doi: 10.1128/JVI.00888-06

Pak, V., Heidecker, G., Pathak, V. K., and Derse, D. (2011). The role of aminoterminal sequences in cellular localization and antiviral activity of APOBEC3B. J. Virol. 85, 8538-8547. doi: 10.1128/JVI.02645-10

Pathak, V. K., and Temin, H. M. (1990). Broad spectrum of in vivo forward mutations, hypermutations, and mutational hotspots in a retroviral shuttle vector after a single replication cycle: substitutions, frameshifts, and hypermutations. Proc. Natl. Acad. Sci. U.S.A. 87, 6019-6023. doi: 10.1073/pnas.87. 16.6019

Pery, E., Rajendran, K. S., Brazier, A. J., and Gabuzda, D. (2009). Regulation of APOBEC3 proteins by a novel YXXL motif in human immunodeficiency virus type 1 Vif and simian immunodeficiency virus SIVagm Vif. J. Virol. 83, 2374-2381. doi: 10.1128/JVI.01898-08

Rahm, N., and Telenti, A. (2012). The role of tripartite motif family members in mediating susceptibility to HIV-1 infection. Curr. Opin. HIV AIDS 7, 180-186. doi: 10.1097/COH.0b013e32835048e1

Rathore, A., Carpenter, M. A., Demir, O., Ikeda, T., Li, M., Shaban, N. M., et al. (2013). The local dinucleotide preference of APOBEC3G can be altered from $5^{\prime}$-CC to $5^{\prime}$-TC by a single amino acid substitution. J. Mol. Biol. 425, 4442-4454. doi: 10.1016/j.jmb.2013.07.040

Refsland, E. W., Hultquist, J. F., and Harris, R. S. (2012). Endogenous origins of HIV-1 G-to-A hypermutation and restriction in the nonpermissive $\mathrm{T}$ cell line CEM2n. PLoS Pathog. 8:e1002800. doi: 10.1371/journal.ppat.1002800

Refsland, E. W., Stenglein, M. D., Shindo, K., Albin, J. S., Brown, W. L., and Harris, R. S. (2010). Quantitative profiling of the full APOBEC3 mRNA repertoire in lymphocytes and tissues: implications for HIV-1 restriction. Nucleic Acids Res. 38, 4274-4284. doi: 10.1093/nar/gkq174

Richardson, S. R., Narvaiza, I., Planegger, R. A., Weitzman, M. D., and Moran, J. V. (2014). APOBEC3A deaminates transiently exposed single-strand DNA during LINE-1 retrotransposition. Elife 3, e02008. doi: 10.7554/eLife.02008

Russell, R. A., Moore, M. D., Hu, W. S., and Pathak, V. K. (2009a). APOBEC3G induces a hypermutation gradient: purifying selection at multiple steps during HIV-1 replication results in levels of G-to-A mutations that are high in DNA, intermediate in cellular viral RNA, and low in virion RNA. Retrovirology 6, 16. doi: 10.1186/1742-4690-6-16

Russell, R. A., Smith, J., Barr, R., Bhattacharyya, D., and Pathak, V. K. (2009b). Distinct domains within APOBEC3G and APOBEC3F interact with separate regions of human immunodeficiency virus type 1 Vif. J. Virol. 83, 1992-2003. doi: 10.1128/JVI.01621-08

Russell, R. A., and Pathak, V. K. (2007). Identification of two distinct human immunodeficiency virus type 1 Vif determinants critical for interactions with human APOBEC3G and APOBEC3F. J. Virol. 81, 8201-8210. doi: 10.1128/JVI. 00395-07

Sadler, H. A., Stenglein, M. D., Harris, R. S., and Mansky, L. M. (2010). APOBEC3G contributes to HIV-1 variation through sublethal mutagenesis. J. Virol. 84, 73967404. doi: 10.1128/JVI.00056-10

Sakai, H., Shibata, R., Sakuragi, J., Sakuragi, S., Kawamura, M., and Adachi, A. (1993). Cell-dependent requirement of human immunodeficiency virus type 1 Vif protein for maturation of virus particles. J. Virol. 67, 1663-1666.

Santa-Marta, M., Da Silva, F. A., Fonseca, A. M., and Goncalves, J. (2005). HIV-1 Vif can directly inhibit apolipoprotein B mRNA-editing enzyme catalytic polypeptide-like 3G-mediated cytidine deamination by using a single amino acid interaction and without protein degradation. J. Biol. Chem. 280, 8765-8775. doi: 10.1074/jbc.M409309200

Schrofelbauer, B., Chen, D., and Landau, N. R. (2004). A single amino acid of APOBEC3G controls its species-specific interaction with virion infectivity factor (Vif). Proc. Natl. Acad. Sci. U.S.A. 101, 3927-3932. doi: 10.1073/pnas.0307132101 Schumacher, A. J., Hache, G., Macduff, D. A., Brown, W. L., and Harris, R. S. (2008). The DNA deaminase activity of human APOBEC3G is required for Ty1, MusD, and human immunodeficiency virus type 1 restriction. J. Virol. 82, 2652-2660. doi: 10.1128/JVI.02391-07

Senavirathne, G., Jaszczur, M., Auerbach, P. A., Upton, T. G., Chelico, L., Goodman, M. F., et al. (2012). Single-stranded DNA scanning and deamination by APOBEC3G cytidine deaminase at single molecule resolution. J. Biol. Chem. 287, 15826-15835. doi: 10.1074/jbc.M112.342790

Shaw, G. M., and Hunter, E. (2012). HIV transmission. Cold Spring Harb. Perspect. Med. 2. doi: $10.1101 /$ cshperspect.a006965 
Sheehy, A. M., Gaddis, N. C., Choi, J. D., and Malim, M. H. (2002). Isolation of a human gene that inhibits HIV-1 infection and is suppressed by the viral Vif protein. Nature 418, 646-650. doi: 10.1038/nature00939

Sheehy, A. M., Gaddis, N. C., and Malim, M. H. (2003). The antiretroviral enzyme APOBEC3G is degraded by the proteasome in response to HIV-1 Vif. Nat. Med. 9, 1404-1407. doi: 10.1038/nm945

Shlyakhtenko, L. S., Lushnikov, A. J., Li, M., Harris, R. S., and Lyubchenko, Y. L. (2014). Interaction of APOBEC3A with DNA Assessed by Atomic Force Microscopy. PLoS ONE 9:e99354. doi: 10.1371/journal.pone.0099354

Shlyakhtenko, L. S., Lushnikov, A. Y., Li, M., Lackey, L., Harris, R. S., and Lyubchenko, Y. L. (2011). Atomic force microscopy studies provide direct evidence for dimerization of the HIV restriction factor APOBEC3G. J. Biol. Chem. 286, 3387-3395. doi: 10.1074/jbc.M110.195685

Shlyakhtenko, L. S., Lushnikov, A. Y., Miyagi, A., Li, M., Harris, R. S., and Lyubchenko, Y. L. (2012). Nanoscale structure and dynamics of ABOBEC3G complexes with single-stranded DNA. Biochemistry 51, 6432-6440. doi: 10.1021/bi300733d

Simon, J. H., Gaddis, N. C., Fouchier, R. A., and Malim, M. H. (1998). Evidence for a newly discovered cellular anti-HIV-1 phenotype. Nat. Med. 4, 1397-1400. doi: $10.1038 / 3987$

Simon, V., Zennou, V., Murray, D., Huang, Y., Ho, D. D., and Bieniasz, P. D. (2005) Natural variation in Vif: differential impact on APOBEC3G/3F and a potential role in HIV-1 diversification. PLoS Pathog. 1:e6. doi: 10.1371/journal.ppat. 0010006

Siu, K. K., Sultana, A., Azimi, F. C., and Lee, J. E. (2013). Structural determinants of HIV-1 Vif susceptibility and DNA binding in APOBEC3F. Nat. Commun. 4, 2593. doi: $10.1038 /$ ncomms3593

Smith, J. L., and Pathak, V. K. (2010). Identification of specific determinants of human APOBEC3F, APOBEC3C, and APOBEC3DE and African green monkey APOBEC3F that interact with HIV-1 Vif. J. Virol. 84, 12599-12608. doi: 10.1128/JVI.01437-10

Song, C., Sutton, L., Johnson, M. E., D’Aquila, R. T., and Donahue, J. P. (2012). Signals in APOBEC3F N-terminal and C-terminal deaminase domains each contribute to encapsidation in HIV-1 virions and are both required for HIV-1 restriction. J. Biol. Chem. 287, 16965-16974. doi: 10.1074/jbc.M111.310839

Soros, V. B., Yonemoto, W., and Greene, W. C. (2007). Newly synthesized APOBEC3G is incorporated into HIV virions, inhibited by HIV RNA, and subsequently activated by RNase H. PLoS Pathog. 3:e15. doi: 10.1371/journal.ppat.0030015

Stanley, B. J., Ehrlich, E. S., Short, L., Yu, Y., Xiao, Z., Yu, X. F., et al. (2008) Structural insight into the human immunodeficiency virus Vif SOCS box and its role in human E3 ubiquitin ligase assembly. J. Virol. 82, 8656-8663. doi: 10.1128/JVI.00767-08

Stopak, K., De Noronha, C., Yonemoto, W., and Greene, W. C. (2003). HIV-1 Vif blocks the antiviral activity of APOBEC3G by impairing both its translation and intracellular stability. Mol. Cell. 12, 591-601. doi: 10.1016/S1097-2765(03) 00353-8

Stopak, K. S., Chiu, Y. L., Kropp, J., Grant, R. M., and Greene, W. C. (2007). Distinct patterns of cytokine regulation of APOBEC3G expression and activity in primary lymphocytes, macrophages, and dendritic cells. J. Biol. Chem. 282, 3539-3546. doi: 10.1074/jbc.M610138200

Strebel, K., Daugherty, D., Clouse, K., Cohen, D., Folks, T., and Martin, M. A. (1987). The HIV 'A' (sor) gene product is essential for virus infectivity. Nature 328, 728-730. doi: 10.1038/328728a0

Strebel, K., and Khan, M. A. (2008). APOBEC3G encapsidation into HIV-1 virions: which RNA is it? Retrovirology 5, 55. doi: 10.1186/1742-4690-5-55

Suspene, R., Rusniok, C., Vartanian, J. P., and Wain-Hobson, S. (2006). Twin gradients in APOBEC3 edited HIV-1 DNA reflect the dynamics of lentiviral replication. Nucleic Acids Res. 34, 4677-4684. doi: 10.1093/nar/gkl555

Suspene, R., Sommer, P., Henry, M., Ferris, S., Guetard, D., Pochet, S., et al. (2004) APOBEC3G is a single-stranded DNA cytidine deaminase and functions independently of HIV reverse transcriptase. Nucleic Acids Res. 32, 2421-2429. doi: 10.1093/nar/gkh554

Svarovskaia, E. S., Xu, H., Mbisa, J. L., Barr, R., Gorelick, R. J., Ono, A., etal. (2004). Human apolipoprotein B mRNA-editing enzyme-catalytic polypeptide-like 3G (APOBEC3G) is incorporated into HIV-1 virions through interactions with viral and nonviral RNAs. J. Biol. Chem. 279, 35822-35828. doi: 10.1074/jbc.M405761200
Tan, L., Sarkis, P. T., Wang, T., Tian, C., and Yu, X. F. (2009). Sole copy of Z2-type human cytidine deaminase APOBEC3H has inhibitory activity against retrotransposons and HIV-1. FASEB J. 23, 279-287. doi: 10.1096/fj.07088781

Tian, C., Yu, X., Zhang, W., Wang, T., Xu, R., and Yu, X. F. (2006). Differential requirement for conserved tryptophans in human immunodeficiency virus type 1 Vif for the selective suppression of APOBEC3G and APOBEC3F. J. Virol. 80, 3112-3115. doi: 10.1128/JVI.80.6.3112-3115.2006

Vartanian, J. P., Henry, M., and Wain-Hobson, S. (2002). Sustained G->A hypermutation during reverse transcription of an entire human immunodeficiency virus type 1 strain Vau group O genome. J. Gen. Virol. 83, 801-805.

Vartanian, J. P., Meyerhans, A., Asjo, B., and Wain-Hobson, S. (1991). Selection, recombination, and $\mathrm{G} \rightarrow \mathrm{A}$ hypermutation of human immunodeficiency virus type 1 genomes. J. Virol. 65, 1779-1788.

Vazquez-Perez, J. A., Ormsby, C. E., Hernandez-Juan, R., Torres, K. J., and Reyes-Teran, G. (2009). APOBEC3G mRNA expression in exposed seronegative and early stage HIV infected individuals decreases with removal of exposure and with disease progression. Retrovirology 6, 23. doi: 10.1186/17424690-6-23

von Hippel, P. H., and Berg, O. G. (1989). Facilitated target location in biological systems. J. Biol. Chem. 264, 675-678.

von Schwedler, U., Song, J., Aiken, C., and Trono, D. (1993). Vif is crucial for human immunodeficiency virus type 1 proviral DNA synthesis in infected cells. J. Virol. 67, 4945-4955.

Wang, H., Liu, B., Liu, X., Li, Z., Yu, X. F., and Zhang, W. (2014). Identification of HIV-1 Vif regions required for CBF-beta Interaction and APOBEC3 suppression. PLoS ONE 9:e95738. doi: 10.1371/journal.pone.0095738

Wang, J., Reuschel, E. L., Shackelford, J. M., Jeang, L., Shivers, D. K., Diehl, J. A., et al. (2011a). HIV-1 Vif promotes the G(1)- to S-phase cell-cycle transition. Blood 117, 1260-1269. doi: 10.1182/blood-2010-06-289215

Wang, X., Abudu, A., Son, S., Dang, Y., Venta, P. J., and Zheng, Y. H. (2011b). Analysis of human APOBEC3H haplotypes and anti-human immunodeficiency virus type 1 activity. J. Virol. 85, 3142-3152. doi: 10.1128/JVI.02049-10

Wang, T., Tian, C., Zhang, W., Luo, K., Sarkis, P. T., Yu, L., et al. (2007). 7SL RNA mediates virion packaging of the antiviral cytidine deaminase APOBEC3G. J. Virol. 81, 13112-13124. doi: 10.1128/JVI.00892-07

Wang, T., Tian, C., Zhang, W., Sarkis, P. T., and Yu, X. F. (2008). Interaction with 7SL RNA but not with HIV-1 genomic RNA or P bodies is required for APOBEC3F virion packaging. J. Mol. Biol. 375, 1098-1112. doi: 10.1016/j.jmb.2007.11.017

Wang, X., Ao, Z., Chen, L., Kobinger, G., Peng, J., and Yao, X. (2012). The cellular antiviral protein APOBEC3G interacts with HIV-1 reverse transcriptase and inhibits its function during viral replication. J. Virol. 86, 3777-3786. doi: 10.1128/JVI.06594-11

Wang, X., Zhang, H., Lv, M., Zuo, T., Wu, H., Wang, J., et al. (2013). Interactions between HIV-1 Vif and human ElonginB-ElonginC are important for CBF-beta binding to Vif. Retrovirology 10, 94. doi: 10.1186/1742-4690-10-94

Wichroski, M. J., Robb, G. B., and Rana, T. M. (2006). Human retroviral host restriction factors APOBEC3G and APOBEC3F localize to mRNA processing bodies. PLoS Pathog. 2:e41. doi: 10.1371/journal.ppat.0020041

Wiegand, H. L., Doehle, B. P., Bogerd, H. P., and Cullen, B. R. (2004). A second human antiretroviral factor, APOBEC3F, is suppressed by the HIV-1 and HIV-2 Vif proteins. EMBO J. 23, 2451-2458. doi: 10.1038/sj.emboj.7600246

Xiao, Z., Ehrlich, E., Luo, K., Xiong, Y., and Yu, X. F. (2007). Zinc chelation inhibits HIV Vif activity and liberates antiviral function of the cytidine deaminase APOBEC3G. FASEB J. 21, 217-222. doi: 10.1096/fj.06-6773com

Xiao, Z., Ehrlich, E., Yu, Y., Luo, K., Wang, T., Tian, C., et al. (2006). Assembly of HIV-1 Vif-Cul5 E3 ubiquitin ligase through a novel zinc-binding domain-stabilized hydrophobic interface in Vif. Virology 349, 290-299. doi: 10.1016/j.virol.2006.02.002

Xu, H., Chertova, E., Chen, J., Ott, D. E., Roser, J. D., Hu, W. S., et al. (2007). Stoichiometry of the antiviral protein APOBEC3G in HIV-1 virions. Virology 360, 247-256. doi: 10.1016/j.virol.2006.10.036

Xu, H., Svarovskaia, E. S., Barr, R., Zhang, Y., Khan, M. A., Strebel, K., et al. (2004). A single amino acid substitution in human APOBEC3G antiretroviral enzyme confers resistance to HIV-1 virion infectivity factor-induced depletion. Proc. Natl. Acad. Sci. U.S.A. 101, 5652-5657. doi: 10.1073/pnas.0400830101

Xu, H., Wang, X., and Veazey, R. S. (2013). Mucosal immunology of HIV infection. Immunol. Rev. 254, 10-33. doi: 10.1111/imr.12072 
Yamashita, T., Kamada, K., Hatcho, K., Adachi, A., and Nomaguchi, M. (2008). Identification of amino acid residues in HIV-1 Vif critical for binding and exclusion of APOBEC3G/F. Microbes Infect. 10, 1142-1149. doi: 10.1016/j.micinf.2008. 06.003

Yang, B., Chen, K., Zhang, C., Huang, S., and Zhang, H. (2007). Virion-associated uracil DNA glycosylase-2 and apurinic/apyrimidinic endonuclease are involved in the degradation of APOBEC3G-edited nascent HIV-1 DNA. J. Biol. Chem. 282, 11667-11675. doi: 10.1074/jbc.M606864200

Yang, X., Goncalves, J., and Gabuzda, D. (1996). Phosphorylation of Vif and its role in HIV-1 replication. J. Biol. Chem. 271, 10121-10129. doi: 10.1074/jbc.271.17.10121

Yu, Q., Konig, R., Pillai, S., Chiles, K., Kearney, M., Palmer, S., et al. (2004). Single-strand specificity of APOBEC3G accounts for minus-strand deamination of the HIV genome. Nat. Struct. Mol. Biol. 11, 435-442. doi: 10.1038/ nsmb758

Yu, X., Yu, Y., Liu, B., Luo, K., Kong, W., Mao, P., et al. (2003). Induction of APOBEC3G ubiquitination and degradation by an HIV-1 Vif-Cul5-SCF complex. Science 302, 1056-1060. doi: 10.1126/science.1089591

Zennou, V., and Bieniasz, P. D. (2006). Comparative analysis of the antiretroviral activity of APOBEC3G and APOBEC3F from primates. Virology 349, 31-40. doi: 10.1016/j.virol.2005.12.035

Zhang, H., Yang, B., Pomerantz, R. J., Zhang, C., Arunachalam, S. C., and Gao, L. (2003). The cytidine deaminase CEM15 induces hypermutation in newly synthesized HIV-1 DNA. Nature 424, 94-98. doi: 10.1038/nature 01707

Zhang, W., Du, J., Evans, S. L., Yu, Y., and Yu, X. F. (2012). T-cell differentiation factor CBF-beta regulates HIV-1 Vif-mediated evasion of host restriction. Nature 481, 376-379. doi: 10.1038/nature10718

Zhen, A., Du, J., Zhou, X., Xiong, Y., and Yu, X. F. (2012). Reduced APOBEC3H variant anti-viral activities are associated with altered RNA binding activities. PLoS ONE 7:e38771. doi: 10.1371/journal.pone.0038771
Zhen, A., Wang, T., Zhao, K., Xiong, Y., and Yu, X. F. (2010). A single amino acid difference in human APOBEC3H variants determines HIV-1 Vif sensitivity. J. Virol. 84, 1902-1911. doi: 10.1128/JVI.01509-09

Zheng, Y. H., Irwin, D., Kurosu, T., Tokunaga, K., Sata, T., and Peterlin, B. M. (2004). Human APOBEC3F is another host factor that blocks human immunodeficiency virus type 1 replication. J. Virol. 78, 6073-6076. doi: 10.1128/JVI.78.11.60736076.2004

Zhou, X., Evans, S. L., Han, X., Liu, Y., and Yu, X. F. (2012). Characterization of the interaction of full-length HIV-1 Vif protein with its key regulator CBFbeta and CRL5 E3 ubiquitin ligase components. PLoS ONE 7:e33495. doi: 10.1371/journal.pone.0033495

Zuo, T., Liu, D., Lv, W., Wang, X., Wang, J., Lv, M., et al. (2012). Smallmolecule inhibition of human immunodeficiency virus type 1 replication by targeting the interaction between Vif and ElonginC. J. Virol. 86, 5497-5507. doi: 10.1128/JVI.06957-11

Conflict of Interest Statement: The authors declare that the research was conducted in the absence of any commercial or financial relationships that could be construed as a potential conflict of interest.

Received: 13 June 2014; accepted: 06 August 2014; published online: 26 August 2014. Citation: Feng Y, Baig TT, Love RP and Chelico L (2014) Suppression of APOBEC3-mediated restriction of HIV-1 by Vif. Front. Microbiol. 5:450. doi: 10.3389/fmicb.2014.00450

This article was submitted to Virology, a section of the journal Frontiers in Microbiology. Copyright (C) 2014 Feng, Baig, Love and Chelico. This is an open-access article distributed under the terms of the Creative Commons Attribution License (CC BY). The use, distribution or reproduction in other forums is permitted, provided the original author(s) or licensor are credited and that the original publication in this journal is cited, in accordance with accepted academic practice. No use, distribution or reproduction is permitted which does not comply with these terms. 